\title{
Large-eddy simulation of a diurnal cycle of the atmospheric boundary layer: Atmospheric stability and scaling issues
}

\author{
Vijayant Kumar, ${ }^{1}$ Jan Kleissl, ${ }^{1,2}$ Charles Meneveau, ${ }^{3}$ and Marc B. Parlange ${ }^{1,4}$ \\ Received 8 October 2005; revised 24 April 2006; accepted 28 April 2006; published 23 June 2006.
}

[1] A simulation of a diurnal cycle of atmospheric boundary layer (ABL) flow over a homogeneous terrain is performed using large-eddy simulation (LES) with the Lagrangian scale-dependent dynamic subgrid-scale model. The surface boundary condition is derived from the field observations of surface heat flux from the HATS experiment (Horst et al., 2004; Kleissl et al., 2004). The simulation results display good general agreement with previous modeling and experimental studies with regard to characteristic features such as growth of the convective boundary layer by entrainment, nocturnal jet, and multilayered flow structure of the nocturnal regime. To gain a better understanding of the physical parameters affecting the statistics of the flow, we study the dependence of a subgrid parameter (dynamic Smagorinsky coefficient), resolved turbulent kinetic energy, and resolved vertical velocity variance upon atmospheric stability. The profiles of these turbulent variables plotted as a function of Obukhov length show "hysteretic" behavior that implies nonunique dependence. The subsequent use of local Richardson number as the scaling parameter shows a decrease in this "hysteresis," but there is an increased scatter in the profiles with increasing height. Conversely, profiles plotted as a function of local Obukhov length (based on the fluxes at the local vertical level) show almost no hysteresis, confirming the validity of Nieuwstadt's local scaling hypothesis. Although the local scaling hypothesis was formulated for the stable boundary layer, we find that it applies to the entire stability range of the diurnal cycle.

Citation: Kumar, V., J. Kleissl, C. Meneveau, and M. B. Parlange (2006), Large-eddy simulation of a diurnal cycle of the atmospheric boundary layer: Atmospheric stability and scaling issues, Water Resour. Res., 42, W06D09, doi:10.1029/2005WR004651.

\section{Introduction}

[2] The diurnal variability in the solar forcing has profound implications on the mass, momentum and energy exchanges occurring in the atmospheric boundary layer (ABL). Since the ABL is turbulent, major turbulence modeling efforts have been directed toward a better understanding of the diurnal structure of atmospheric turbulence. Yamada and Mellor [1975] and André et al. [1978] modeled the diurnal structure of the ABL with Reynolds-averaged Navier-Stokes (RANS) methodology using second- and third-order turbulence closure models, respectively. The RANS approach is focused on the evolution of mean quantities and the effect of the unsteadiness in the flow due to turbulence is accounted for by a turbulence closure

\footnotetext{
${ }^{1}$ Department of Geography and Environmental Engineering and Center for Environmental and Applied Fluid Mechanics, Johns Hopkins University, Baltimore, Maryland, USA.

${ }^{2}$ Now at Department of Earth and Environmental Sciences, New Mexico Institute of Mining and Technology, Socorro, New Mexico, USA.

${ }^{3}$ Department of Mechanical Engineering and Center for Environmental and Applied Fluid Mechanics, Johns Hopkins University, Baltimore, Maryland, USA.

${ }^{4}$ School of Architecture, Civil and Environmental Engineering, Ecole Polytechnique Fédérale de Lausanne, Lausanne, Switzerland.

Copyright 2006 by the American Geophysical Union. 0043-1397/06/2005WR004651\$09.00
}

model. Around the same time, pioneering works by Deardorff [1972, 1974] led to advent of large-eddy simulation (LES) technique for meteorological applications. The LES technique explicitly simulates the large-scale structures resolved by the computational grid while the effects of the small, unresolved scales are parameterized using a subgrid-scale (SGS) model. LES has emerged as a popular technique for simulation of ABL flows in idealized neutral, convective (daytime) and stable (nocturnal) atmospheric boundary layers [Moeng, 1984; Andrén et al., 1994; Nieuwstadt et al., 1991; Kosović and Curry, 2000].

[3] LES studies of the convective boundary layer have been largely successful mainly due to the dominance of large-scale structures in the flow [Schmidt and Schumann, 1989; Nieuwstadt et al., 1991]. However, several LES studies have reported strong departures of velocity and temperature spectra from the expected inertial scaling and these departures have been usually attributed to the sensitivity and often overdissipative nature of SGS models [Khanna and Brasseur, 1997; Nieuwstadt et al., 1991]. While LES studies of convective ABL have been performed successfully for the last 25 years, LES has begun to be applied to stable boundary layers only recently. Even the LES studies of quasi-steady stable boundary layers (i.e., stable boundary layers with an imposed constant surface heat flux or constant cooling) have reported problems associated with poor SGS models, low grid resolutions 
and numerical instabilities [Beare and MacVean, 2004; Derbyshire, 1999]. For example, Mason and Derbyshire [1990] attributed failures in LES of a strongly cooled $\left(\overline{w^{\prime} \theta^{\prime}} \sim-0.03 \mathrm{Kms}^{-1}\right)$ stable boundary layer to the SGS model. Saiki et al. [2000] reported numerical instabilities when the heat flux changed sign (from positive to negative) and attributed it to the numerics and the SGS model. While Mason and Derbyshire [1990] used a Smagorinsky model with wall damping and stability correction functions, Saiki et al. [2000] used a modified twopart SGS model.

[4] It is important to note that most research on the ABL with LES is based on simulations of quasi-steady ABL flows (with constant surface heat flux/cooling), but this is never the case in the real-world ABL. A critical test of the performance of LES is the simulation of the diurnal cycle of the ABL. The difficulties associated with simulating an entire diurnal cycle of the ABL stem from the extreme disparities between the nature of the two main parts of the diurnal ABL: the daytime convective boundary layer $(\mathrm{CBL})$ and the nocturnal stable boundary layer (SBL). The structure of the CBL is characterized by largescale plumes and production of turbulent kinetic energy by shear and buoyancy, leading to enhanced mixing of mass, momentum and energy. The SBL is instead marked by significantly smaller turbulent structures, strong nearsurface inversions, radiative cooling and competition between mechanical production and buoyant destruction of turbulent kinetic energy. To add to the complexity, the simulations also need to accurately represent the less understood morning and evening transition regimes from the SBL to the CBL and vice versa.

[5] There have been some applications of LES to the study of unsteady ABL evolution. Nieuwstadt and Brost [1986] performed a highly idealized study of the decay of convective turbulence by initializing an LES run with a quasi-steady CBL field and studying its evolution with the heat flux set to 0. Sorbjan [1997] extended this CBL study using gradually decaying heat flux with zero geostrophic wind. An LES intercomparison study of daytime shallow cumulus convection over land was performed by Brown et al. [2002] in which the sensible heat flux, derived from the 1997 Southern Great Plains experiment was used as the surface boundary condition. Brown et al. [2002] noted the difficulties associated with LES investigations of strong time-dependent forcings and the need for higher-resolution studies of the stable boundary layer. Note that the stable boundary layer regime was not simulated in the study by Brown et al. [2002].

[6] Recently, LES has been applied to the complete diurnal cycle of stratocumulus-topped marine boundary layers [Chlond et al., 2004; Duynkerke et al., 2004]. The focus of both these studies is on understanding the diurnal evolution of stratocumulus and providing benchmarking tools for single-column global circulation models (GCMs). Neither study included a detailed analysis of the turbulent structure of the diurnal ABL over land.

[7] In addition to the aforesaid issues concerning the LES investigations of diurnal ABL, a critical issue is the applicability of the standard scaling parameters (such as Obukhov length and Richardson numbers) across multistability flow regimes. While these scaling parameters have been found to work well for quasi-steady ABL flows [Khanna and Brasseur, 1997], there is an urgent need to study the utility of these parameters in ABLs with temporally varying forcings. For instance, Monin-Obukhov similarity theory which uses Obukhov length as a scaling parameter is widely used in regional and global climatic models.

[8] The issues presented above make the case for LES investigations of the structure of the diurnal ABL and its impact on scaling. The goal of this work therefore is to perform an LES investigation of the diurnal ABL over land with the focus on reproducing its established features and performing a detailed analysis of its turbulent structure. Specifically, we conduct a high-resolution LES of ABL over homogeneous terrain using a state-of-the-art Lagrangian dynamic scale-dependent SGS model [Bou-Zeid et al., 2005] to examine the performance of the LES technique and to examine scaling relationships. The LES technique and SGS parameterizations are presented in section 2 together with details of numerics and simulation setup. In section 3.1, we compare LES results with field observations and in section 3.2, we examine the ability of LES to reproduce well-known features of the diurnal cycle of the ABL. Finally, in sections 3.3 and 3.4, we examine some scaling issues for the variables spanning the entire stability range of the diurnal cycle of the $\mathrm{ABL}$ and the conclusions are summarized in section 4.

\section{Large-Eddy Simulation: Technical Formulation}

[9] The flow dynamics and the transport of mass, momentum and energy in the ABL are analytically represented by the incompressible Navier-Stokes and accompanying set of scalar conservation equations [Landau and Lifshitz, 1959; Businger, 1982]. With the ratio between the largest $(\sim 1 \mathrm{~km})$ and the smallest $(\sim 1 \mathrm{~mm})$ scales of motion in the ABL being approximately $\mathrm{O}\left(10^{6}\right)$, it is currently impossible to resolve all these scales of motions explicitly using available computational resources [Pope, 2000]. Large-eddy simulation alleviates this problem by the use of filtering (i.e., the application of a convolution kernel) wherein all scales larger than the grid scale are explicitly computed while the remaining small scales, i.e., the subgrid scales (SGS), are parameterized using a SGS model [Lesieur and Métais, 1996]. The LES formulation is based on the premise that the large resolved eddies transport most of the mass, momentum and energy and are modified significantly by geometry and nature of the underlying problem. The small SGS eddies on the other hand, are more homogeneous and isotropic in character and are less affected by the boundary conditions than the large scales. Therefore the subgrid scales are more amenable to a universal parameterization. Note that the idea of filtering is fundamentally different from the RANS approach where the equations of motion are averaged with the turbulence making its appearance only in the form of a closure parameterization [Tennekes and Lumley, 1972; Pope, 2000]. The filtered LES field contains turbulent motions and structures spanning a wide range of scales, devoid just of the scales lost by virtue of the filtering. The filtered set of the governing equations for an LES of 

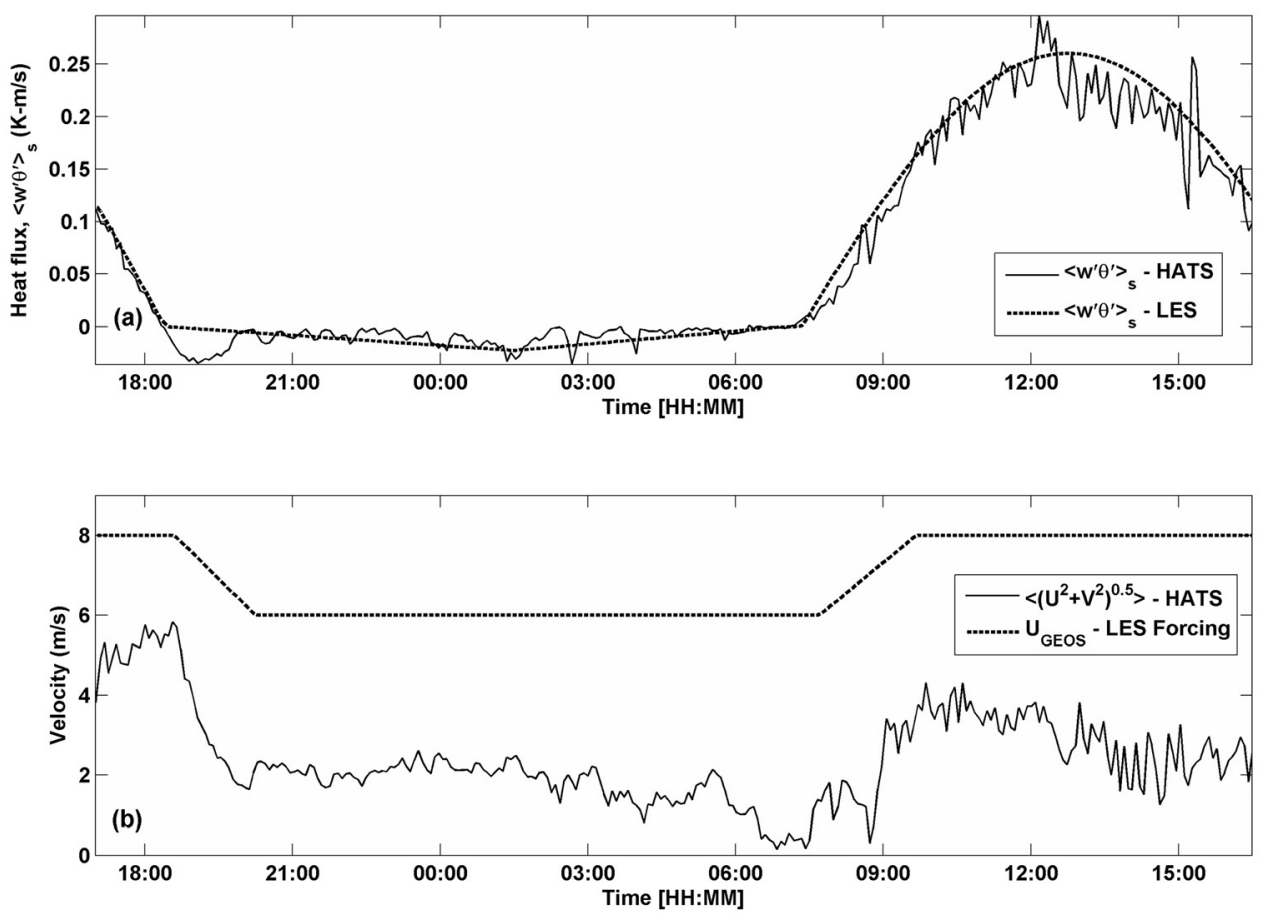

Figure 1. (a) Observed diurnal cycle of surface heat flux in HATS 2000 and the smoothed surface heat flux boundary condition in LES. (b) Variation of geostrophic wind forcing in the LES and the observed variation of near-surface wind velocity in HATS.

incompressible, high Reynolds number $(R e)$ flow in the ABL may be written as

$$
\begin{gathered}
\frac{\partial u_{i}}{\partial x_{i}}=0 \\
\frac{\partial \widetilde{u}_{i}}{\partial t}+\widetilde{u}_{j}\left(\frac{\partial \widetilde{u}_{i}}{\partial x_{j}}-\frac{\partial \widetilde{u}_{j}}{\partial x_{i}}\right)=-\frac{1}{\rho} \frac{\partial \widetilde{p}}{\partial x_{i}}+g\left(\frac{\widetilde{\theta}-\langle\widetilde{\theta}\rangle}{\langle\widetilde{\theta}\rangle}\right) \delta_{i 3} \\
-\frac{\partial \widetilde{\tau}_{i j}}{\partial x_{j}}+f\left(\widetilde{u}_{2}-V_{g}\right) \delta_{i 1} \\
-f\left(\widetilde{u}_{1}-U_{g}\right) \delta_{i 2} \\
\frac{\partial \widetilde{\theta}}{\partial t}+\widetilde{u}_{j} \frac{\partial \widetilde{\theta}}{\partial x_{j}}=-\frac{\partial \pi_{j}}{\partial x_{j}} .
\end{gathered}
$$

Equation (1) is the filtered equation for conservation of mass, equation (2) is the momentum transport equation, i.e., the filtered Navier-Stokes equation in rotational form and includes the Boussinesq approximation, and equation (3) is the governing equation for a conserved scalar (potential temperature, $\theta$ in this case). The rotational form is used for kinetic energy and mass conservation [Orszag and Pao, 1974]. In these equations, a tilde $(\sim)$ denotes a filtered variable (at the grid scale $\Delta$ ), \langle\rangle represents averaging over horizontal planes, $\widetilde{u}_{i}$ is the resolved (filtered) velocity component in the $i$ direction ( $i=1,2,3), f$ is the Coriolis parameter, $U_{g}$ and $V_{g}$ are the horizontal components of the geostrophic wind, $\widetilde{p}$ is the resolved dynamic pressure term formulated so as to satisfy the divergence-free condition, $\tau_{i j}$ is the deviatoric part of the SGS stress tensor, $\widetilde{\theta}$ is the filtered potential temperature, $\pi_{j}$ is the SGS flux of temperature and $\delta_{i j}$ is the Kronecker delta ( $\delta_{i j}=1$ for $i=j, 0$ otherwise). The potential temperature being an active scalar affects the velocity field by creating vertical accelerations and this has been represented with aid of the Boussinesq approximation in equation (2). To achieve closure of the above set of equations (1)-(3), the SGS terms $\tau_{i j}$ and $\pi_{j}$ are respectively parameterized in the form of Smagorinskytype "eddy viscosity" and "eddy diffusivity" closures detailed in section 2.1 .

\subsection{SGS Modeling}

[10] SGS stresses have been most often parameterized through the well-known Smagorinsky model [Smagorinsky, 1963] which is basically the LES representation of Prandtl's mixing length hypothesis expressed as

$$
\tau_{i j}^{S m a g}=-2 \nu_{T} \widetilde{S}_{i j}=-2\left(C_{s, \Delta} \Delta\right)^{2}|\widetilde{S}| \widetilde{S}_{i j}
$$

where $\widetilde{S}_{i j}$ is the resolved strain rate tensor, $\nu_{T}$ is the eddy viscosity $\left(2_{T}=\left(C_{S, \Delta} \Delta\right)^{2}\left|\widetilde{S}_{i j}\right|\right)$ where $C_{S, \Delta}$ is known as the Smagorinsky coefficient. The scalar SGS flux term, $\pi_{j}$ in equation (3), is analogously parameterized as $\pi_{j}=$ - $\left(\nu_{T} / P r_{s g s}\right)\left(\partial \theta / \partial x_{j}\right)$ where $P r_{s g s}$ is the SGS turbulent Prandtl number. Notice that with these parameterizations, the coefficient, $C_{s, \Delta}$ (or $C_{s}$ ) and $P r_{s g s}$ are the only unknown parameters. Historically, $C_{S, \Delta}$ has been fixed a priori in the vicinity of 0.17 , based on the analysis for homogeneous isotropic turbulence [Lilly, 1967]. This estimate works well for simulations of ABL flows over homogeneous terrains, specifically in regions far away from the surface. In the vicinity of the lower boundary, the local integral scale is on the order of the distance 

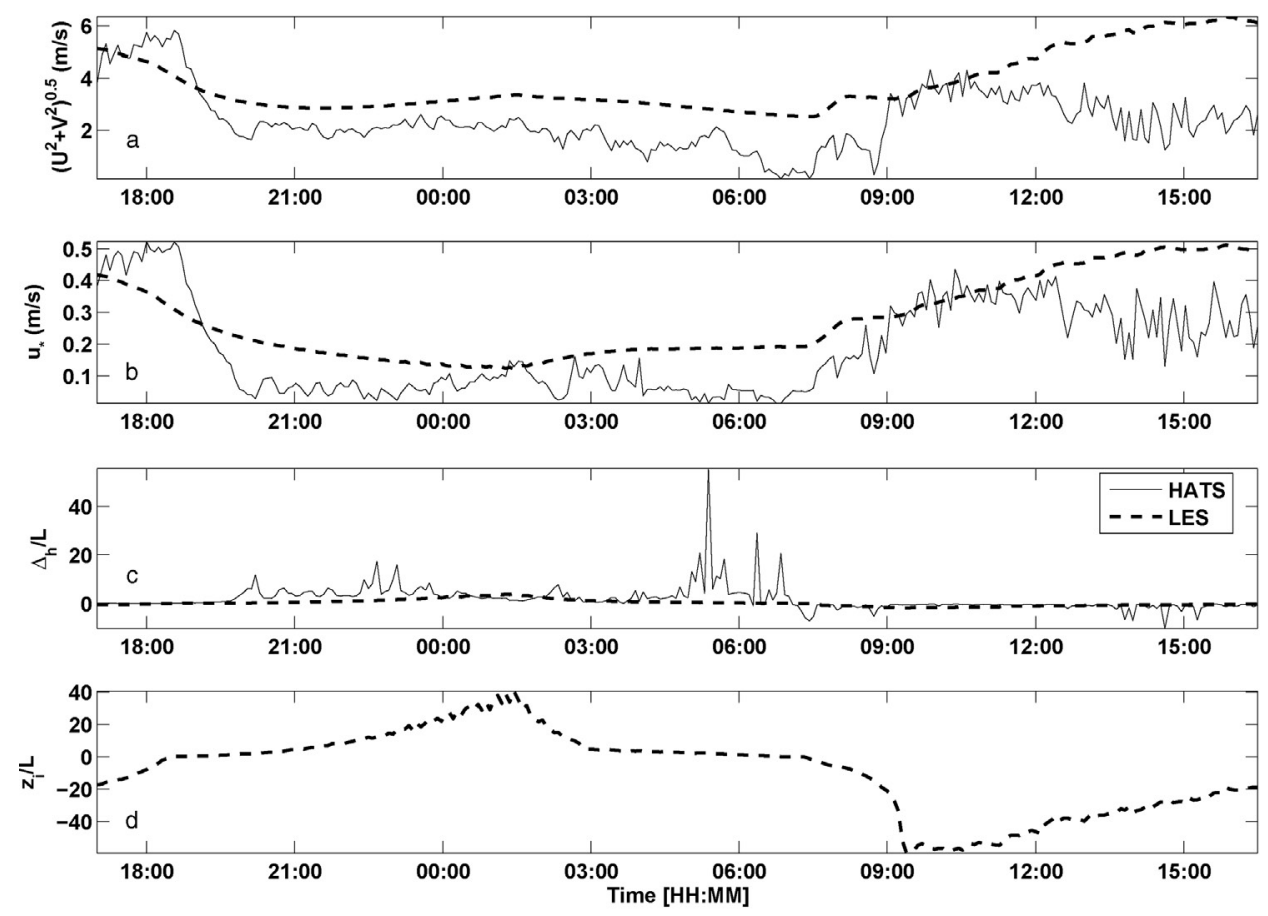

Figure 2. Comparison between LES computed variables and HATS observations of 5 September 2000 (a)horizontal wind velocity, $U_{\text {res }}=\left(\langle U\rangle^{2}+\langle V\rangle^{2}\right)^{0.5}$, (b) friction velocity, $u *$, (c) stability parameter, $\Delta_{h} / L$, and (d) stability parameter, $z_{i} / L$, where $z_{i}$ is the boundary layer height. The thin solid line represents the HATS observations, while the thick solid line represents the LES results.

from the wall and the subgrid scales represent a significantly larger portion of the momentum and scalar fluxes. Here, the a priori estimate fails [Kleissl et al., 2003]. In simulations of ABL flows with temporally varying boundary conditions or over heterogeneous terrain, the application of an SGS model based on a priori estimates is fundamentally flawed as it cannot account for the local and temporal variability of $C_{s}$.

[11] A major paradigm shift in SGS modeling occurred with the development of the "dynamic model" [Germano et al., 1991]. In this pioneering work, the information contained in the resolved fields is used to compute the coefficient dynamically and internally within the LES by virtue of filtering of the resolved fields at two scales, the grid scale, $\Delta$, and the test filter scale, $2 \Delta$.

[12] The original formulation of the dynamic model is based on the assumption of scale invariance $\left(\beta=C_{s, 2 \Delta}^{2} / C_{s, \Delta}^{2}\right.$ $=1$ ). However, this assumption does not hold in the vicinity of the surface [Porté-Agel et al., 2000b] and in stable conditions [Kleissl et al., 2003], and a proper SGS formulation should incorporate scale dependence in such scenarios. In order to account for dependence on scale, Porté-Agel et al. [2000a] introduced the so-called scaledependent dynamic model based on another filter operation at scale $4 \Delta$. Both the original and the scale-dependent formulation of the dynamic model rely on spatial averaging over horizontal planes to compute $C_{s}$. In order to account for spatial nonuniformity and for applications to complex geometry flow, Meneveau et al. [1996] introduced the Lagrangian approach in which $C_{s}$ is determined from time averaging following fluid trajectories instead of traditional spatial averaging. Finally, Bou-Zeid et al.
[2005] have combined both approaches to arrive at the Lagrangian scale-dependent dynamic model. In this model, after replacing the stresses at scales $\Delta, 2 \Delta$ and $4 \Delta$ with the Smagorinsky model, equations for the coefficients $C_{s, \Delta}^{2}$ are attained at various scales. Finally, the position and timedependent model parameter $C_{S}$ can be written according to

$$
C_{s, \Delta}^{2}=\frac{\mathcal{I}_{L M} / \mathcal{I}_{M M}}{\max \left[\frac{\mathcal{I}_{Q N} \mathcal{I}_{M M}}{\mathcal{I}_{N N} \mathcal{I}_{L M}}, 1 / 8\right]} .
$$

The terms $\mathcal{I}_{L M}, \mathcal{I}_{M M}, \mathcal{I}_{Q N}$ and $\mathcal{I}_{N N}$ are quantities obtained from Lagrangian averaging tensor contractions involving stresses and strain rates at scales $\Delta, 2 \Delta$ and $4 \Delta$. For more details regarding the theory, formulation and implementation of the Lagrangian scale-dependent dynamic SGS model, see Bou-Zeid et al. [2005]. This model is particularly well suited for simulations of unsteady flows over inhomogeneous terrain owing to its strengths such as physically motivated path-line-based averaging, preservation of local variability and improved SGS dissipation characteristics in the near-wall region.

[13] The SGS turbulent Prandtl number, $P r_{s g s}$ is prescribed as a constant equal to 0.4 and is based on inertial subrange theory [Mason, 1989], simulations [AntonopoulosDomis, 1981] and observations [Kang and Meneveau, 2002]. Recent experimental evidence obtained in the atmospheric surface layer suggests that $P r_{s g s}$ is weakly dependent on stability and increases weakly with increase in $\Delta / z$ [Kleissl et al., 2003; Porté-Agel et al., 2001]. Scale-dependent formulations for dynamic computation of $P r_{s g s}$ in LES 
Diurnal variation of potential temperature, $\theta(\mathrm{K})$
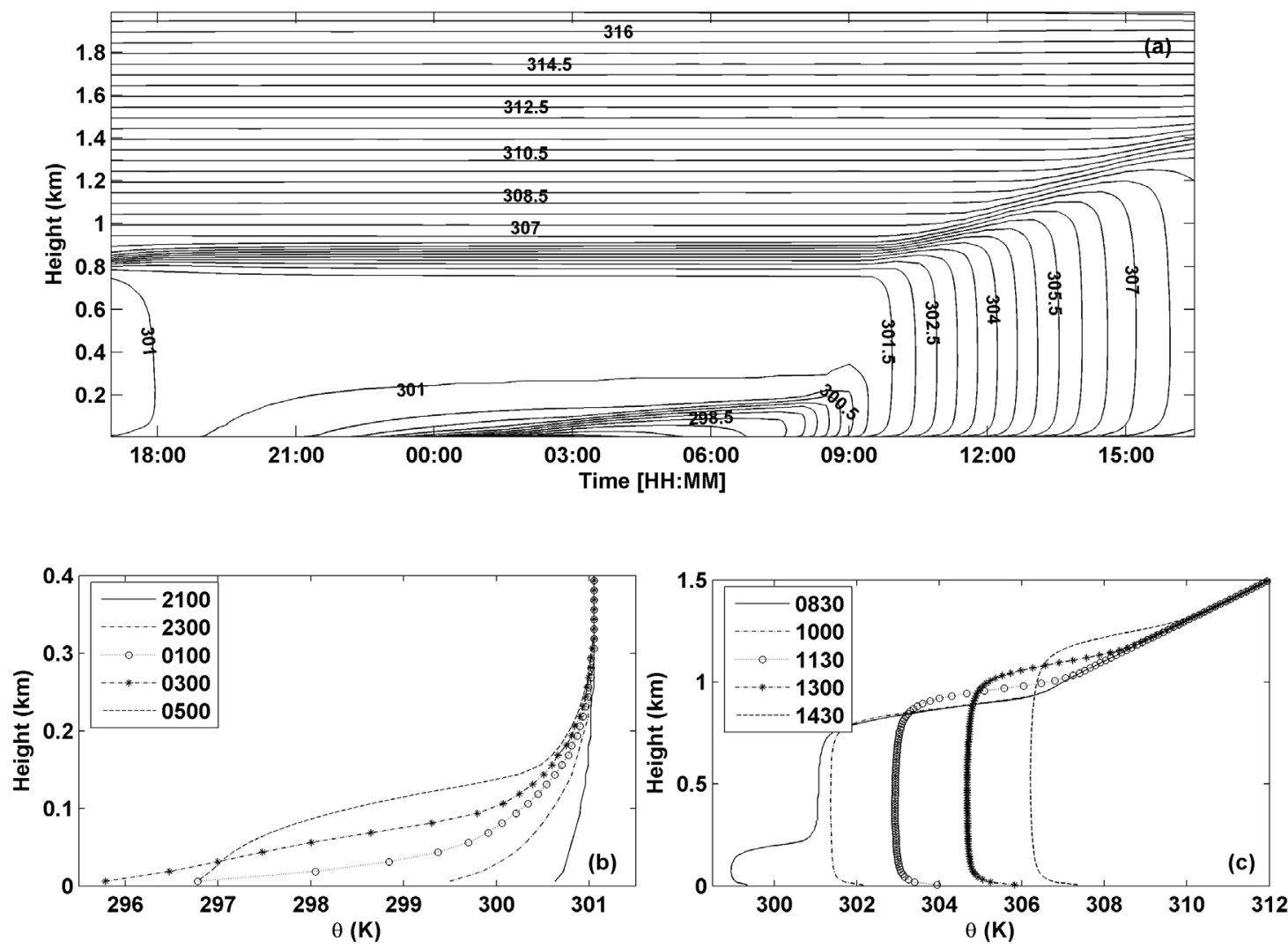

Figure 3. (a) Contour plot of potential temperature, $\widetilde{\theta}(K)$ during the diurnal evolution of ABL. (b) Profiles of potential temperature during the evolution of the stable boundary layer. Notice the growth of the surface inversion over time. (c) Evolution of the convective boundary layer. Notice the increase in the depth of the CBL by entrainment from the overlying inversion layer.

have also been recently developed [Porté-Agel, 2004; Stoll and Porté-Agel, 2006].

\subsection{Description of the LES Code}

[14] The basic LES code used here traces its origins to the work of Albertson and Parlange [1999, 2000]. The code solves the rotational form of the high Re Navier-Stokes equations (equation (3)) in a pseudospectral setup with spectral decomposition in the two horizontal directions and second-order centered finite differences in a staggered grid formulation in the vertical direction. The second-orderaccurate Adams Bashforth method is used for temporal integration [Canuto et al., 1988]. The dealiasing of nonlinear terms is performed in Fourier space by the 3/2 rule [Orszag, 1970]. The code has been modified in the subsequent work of Porté-Agel et al. [2000a] and Bou-Zeid et al. [2004, 2005]. In view of the current trends for modular codes in computational fluid dynamics, the LES code has been completely rewritten using modular features of Fortran 90. In view of the significant advances in available computational power, the code has also been parallelized using horizontal slice-based domain decomposition and MPI (message passing interface). The pressure solver for parallel runs is based on the pipelined Thomas algorithm [Povitsky and Morris, 2000].

[15] The flow is driven by a mean pressure gradient imposed in terms of horizontal geostrophic wind, $\left(U_{g}, V_{g}\right)$ through the use of geostrophic approximation. The lower boundary condition is formulated using the MoninObukhov similarity theory wherein the instantaneous surface stress is represented as a function of instantaneous horizontal velocities, $\widetilde{u}$ and $\widetilde{v}$ at the first grid point $(d z / 2)$ above the surface:

$$
\begin{aligned}
& \tau_{x z}=-u_{*}^{2}\left[\frac{\widehat{\widetilde{u}}}{\sqrt{\widehat{\widetilde{u}}^{2}+\widehat{\widehat{v}}^{2}}}\right] \\
& \tau_{y z}=-u_{*}^{2}\left[\frac{\widehat{\widetilde{v}}}{\sqrt{\widehat{\widetilde{u}}^{2}+\widehat{\widehat{v}}^{2}}}\right]
\end{aligned}
$$

where $u *$ is the friction velocity computed as

$$
u *=\frac{\kappa \sqrt{\widehat{\widetilde{u}}^{2}+\widehat{\widehat{v}}^{2}}}{\ln \left(\frac{z}{z_{0}}\right)-\psi_{m}\left(\frac{z}{L}\right)} .
$$

Here $\kappa=0.4$ is the von Karman constant, $z_{o}$ is the momentum roughness length and $L$ is the Obukhov length. Note that in equations (6)-(8), the instantaneous velocities $\widetilde{u}$ and $\widetilde{v}$ are explicitly test filtered at scale $2 \Delta$ as represented

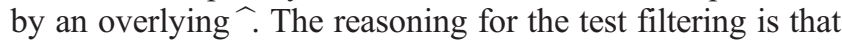
the Monin-Obukhov similarity theory for the mean flow is 

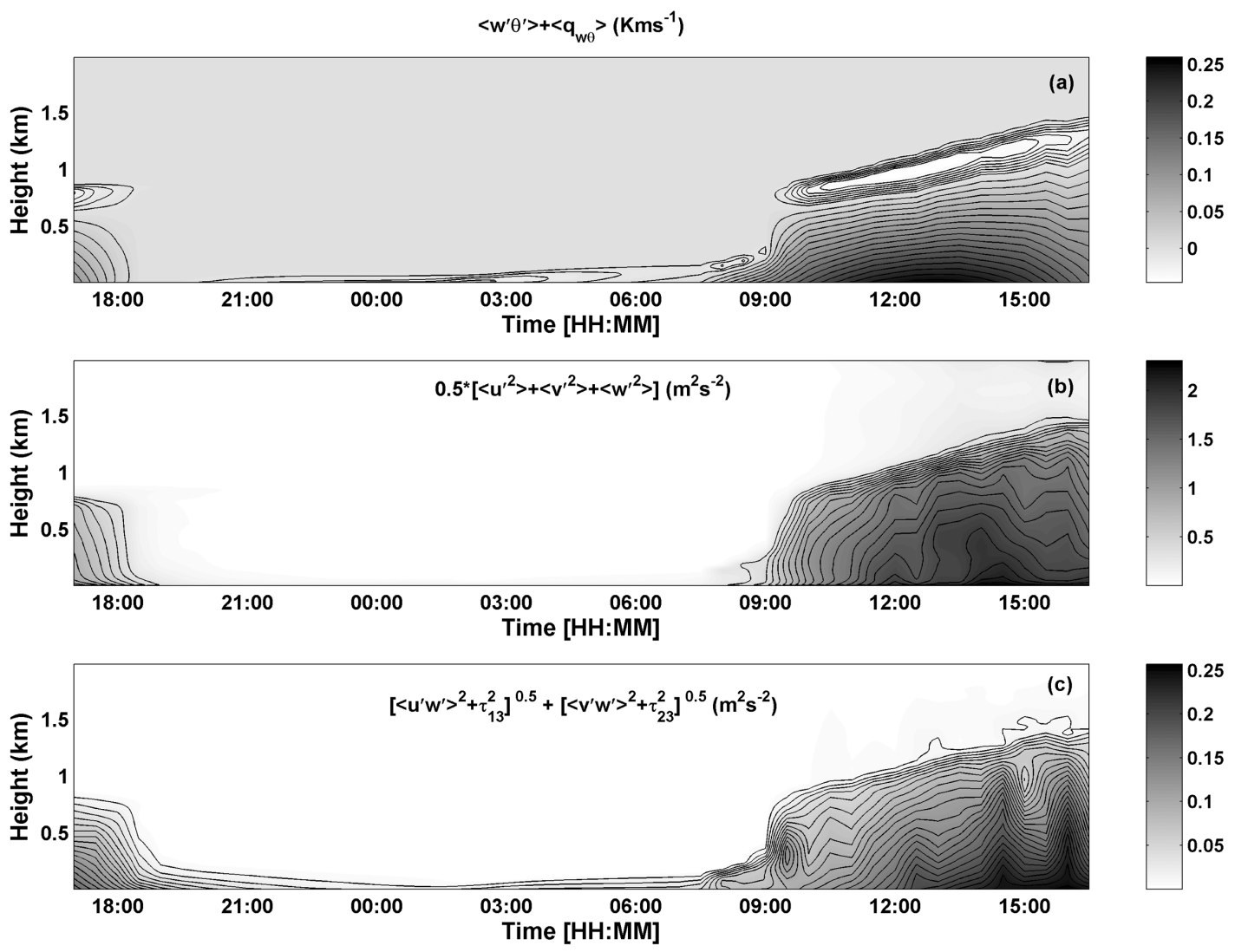

Figure 4. Diurnal evolution of the vertical profile of (a) sensible heat flux, $\left\langle w^{\prime} \theta^{\prime}\right\rangle+\left\langle q_{w \theta}\right\rangle\left(\mathrm{Kms}^{-1}\right)$, (b) resolved turbulent kinetic energy, $q=0.5\left[\left\langle u^{\prime 2}\right\rangle+\left\langle v^{\prime 2}\right\rangle+\left\langle w^{\prime 2}\right\rangle\right]\left(\mathrm{m}^{2} \mathrm{~s}^{-2}\right)$, and (c) total momentum flux, $\left[\left\langle u^{\prime} w^{\prime}\right\rangle^{2}+\tau_{13}^{2}\right]^{0.5}+\left[\left\langle v^{\prime} w^{\prime}\right\rangle^{2}+\tau_{23}^{2}\right]^{0.5}\left(\mathrm{~m}^{2} \mathrm{~s}^{-2}\right)$.
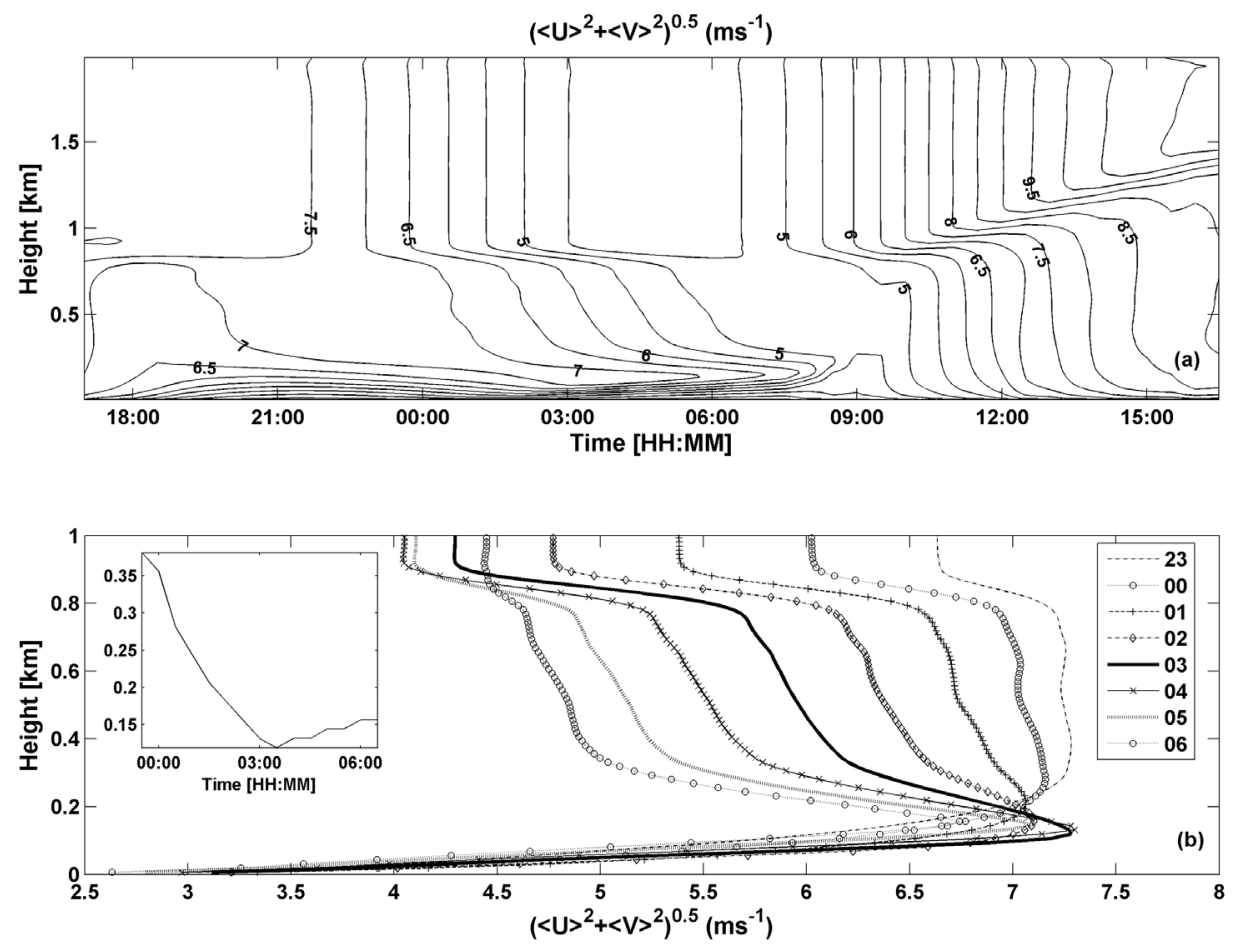

Figure 5. (a) Diurnal evolution of horizontal wind velocity, $\left[\langle U\rangle^{2}+\langle V\rangle^{2}\right]^{0.5}\left(\mathrm{~ms}^{-1}\right)$. (b) Evolution of low-level jet in the stable boundary layer. The legend displays the hour corresponding to the plotted velocity profile. The solid line in the insert traces the height of the LLJ wind maxima across time. 


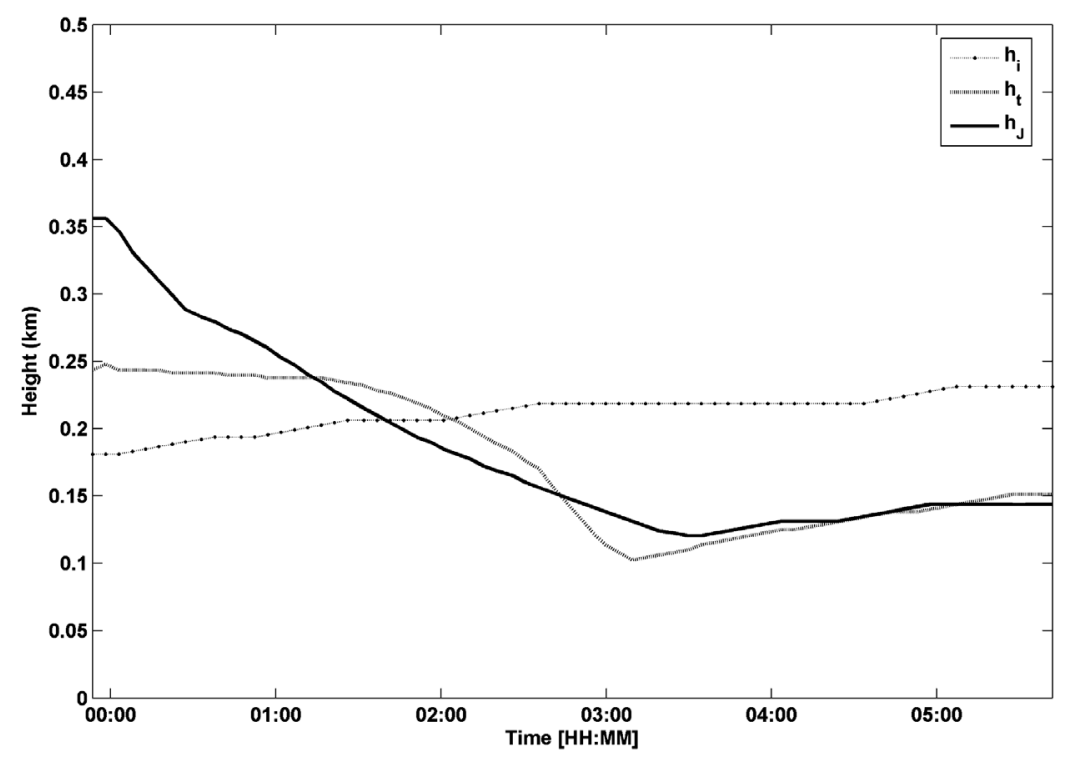

Figure 6. Temporal evolution of the depth of surface inversion layer $h_{i}$, depth of turbulent layer $h_{t}$, and height of low-level jet $h_{J}$ in the nocturnal stable boundary layer.

applied here in a strictly local sense and the test filtering therefore serves to keep the small-scale fluctuations in check while still preserving large-scale variations [Bou-Zeid et al., 2005].

[16] The $\psi_{m}$ functions introduced in equation 8 are defined as $\psi_{m}(z / L)=\int_{z / L}^{z / L}\left[1-\phi_{m}(x)\right] d x / x$. The MoninObukhov stability correction functions for nondimensional gradients $\phi_{m}\left(=-\frac{\kappa z}{u *} \frac{d U}{d z}\right)$ and $\phi_{h}\left(=-\frac{\kappa z u}{\overline{w^{\prime} \theta^{\prime}}} \frac{d \theta}{d z}\right)$ are needed for the bottom boundary condition. For unstable conditions, the formulation of $\phi_{m}$ and $\phi_{h}$ is based on the work of Hogstrom [1987]. For the stable regimes, the simple formulations for $\phi_{m}$ and $\phi_{h}$ of type $\alpha+\beta \frac{z}{L}$ (where $\alpha$ and $\beta$ are constants) are not consistent with observations when $\frac{z}{L}$ is greater than 0.8 [Hicks, 1976; Holtslag and De Bruin,

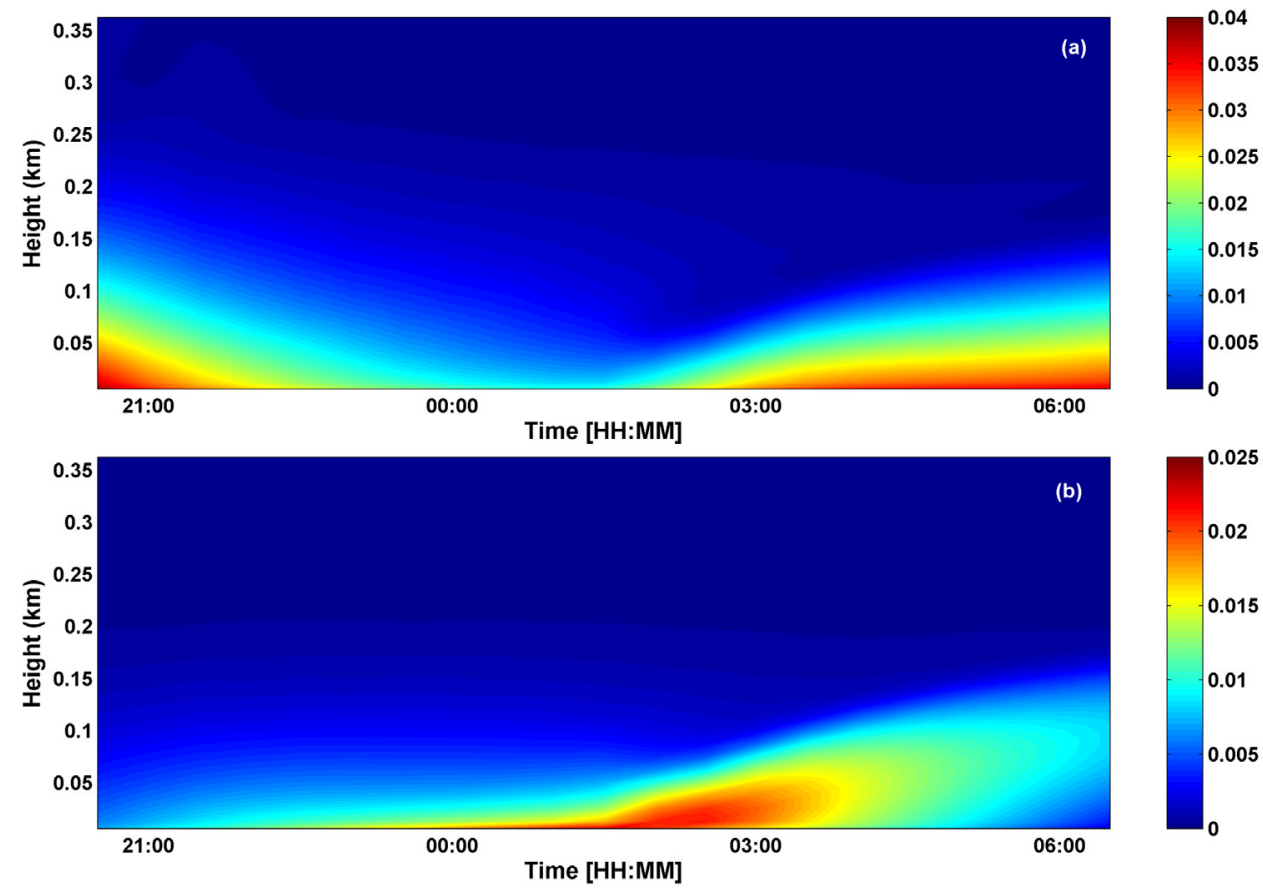

Figure 7. Turbulent flux evolution in the stable, nocturnal regime of the ABL (2100-0600 hours). (a) Total momentum flux, $\left[\left\langle u^{\prime} w^{\prime}\right\rangle^{2}+\tau_{13}^{2}\right]^{0.5}+\left[\left\langle v^{\prime} w^{\prime}\right\rangle^{2}+\tau_{23}^{2}\right]^{0.5}\left(\mathrm{~m}^{2} \mathrm{~s}^{-2}\right)$. (b) Negative of total sensible heat flux, $-\left[\left\langle w^{\prime} \theta^{\prime}\right\rangle+\left\langle q_{w \theta}\right\rangle\right]\left(\mathrm{Kms}^{-1}\right)$. Note that the sign of sensible heat flux has been inverted to keep the coloring pattern consistent across the two plots. 


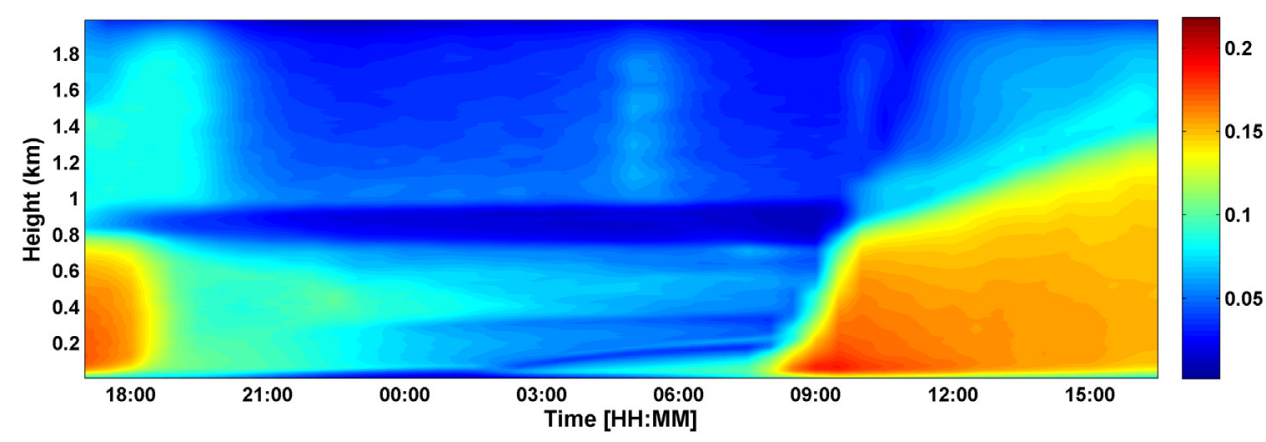

Figure 8. Diurnal evolution of Smagorinsky coefficient, $C_{S}$ (averaged over horizontal planes), using the Lagrangian scale-dependent SGS model.

1988], and hence the modified formulation of Cheng and Brutsaert [2005] is used. Thus $\phi_{m}$ and $\phi_{h}$ are given by

$$
\begin{aligned}
& \phi_{m}=\left\{\begin{array}{cl}
\left(1-15.2 \frac{z}{L}\right)^{-0.25} & : \frac{z}{L}<0 \\
1+6.1 \frac{\frac{z}{L}+\left(\frac{z}{L}\right)^{a}\left(1+\left(\frac{z}{L}\right)^{a}\right)^{[-1+1 / a]}}{\frac{z}{L}+\left(1+\left(\frac{z}{L}\right)^{a}\right)^{1 / a}} & : \frac{z}{L}>0
\end{array}\right. \\
& \phi_{h}=\left\{\begin{array}{cl}
\left(1-15.2 \frac{z}{L}\right)^{-0.5} & : \frac{z}{L}<0 \\
1+5.3 \frac{\frac{z}{L}+\left(\frac{z}{L}\right)^{b}\left(1+\left(\frac{z}{L}\right)^{b}\right)^{[-1+1 / b]}}{\frac{z}{L}+\left(1+\left(\frac{z}{L}\right)^{b}\right)^{1 / b}} & : \frac{z}{L}>0
\end{array}\right.
\end{aligned}
$$

where $a=2.5$ and $b=1.1$. The upper boundary condition for momentum transport consists of a zero-stress condition, while a fixed temperature gradient is used as the top boundary condition for scalar transport. A damping layer is used in the vicinity of the domain top in order to dissipate the energy and thereby, prevent the undesired reflection of gravity waves at the top boundary of the domain [Nieuwstadt et al., 1991].

\section{Simulation of the Diurnal Cycle of ABL}

\subsection{Simulation Setup and Baseline Results}

[17] In order to validate the LES code for nonneutral conditions, simulations of quasi-steady convective and stable boundary layers were performed by Kleissl et al. [2006]. The results show improved performance of the Lagrangian dynamic scale-dependent (LASD) SGS model compared to the Lagrangian dynamic scale-invariant SGS model in computing more accurate values of $C_{s}$, and in accurately representing the inertial range scaling in velocity and scalar spectra. Therefore we adopt the LASD SGS model for the simulation of the diurnal cycle of ABL.

[18] The LES of the diurnal cycle of ABL is performed over a domain of size $4 \mathrm{~km} \times 4 \mathrm{~km} \times 2 \mathrm{~km}$ discretized using 160 nodes in each direction totalling to 4.1 million points. The horizontal resolution is $\Delta_{x}=\Delta_{y}=25 \mathrm{~m}$ while the vertical resolution is $\Delta_{z}=12.5 \mathrm{~m}$. The diurnal simulation is initialized using the data from a 1 hour simulation of a convective boundary layer with a constant surface heat flux, $\overline{w^{\prime} \theta^{\prime}}=0.1 \mathrm{Kms}^{-1}$. The convective boundary layer is capped by an overlying inversion layer with a constant inversion strength of $0.01 \mathrm{Km}^{-1}$.

[19] The data collected during the HATS experiments [Horst et al., 2004; Kleissl et al., 2004] have been used to generate boundary conditions for the simulated diurnal cycle. The heat flux boundary condition is a smoothed version of the 24 hour heat flux data observed on 6 September 2000 during the HATS experiment (Figure 1a). The momentum roughness length at the surface is set to $z_{o}=0.02 \mathrm{~m}$ based on the value determined from the HATS data.

[20] The geostrophic wind is set to $\left(U_{g}, V_{g}\right)=(8,0) \mathrm{ms}^{-1}$ during the daytime part of the day and is gradually decreased to $(6,0) \mathrm{ms}^{-1}$ during the nocturnal regime based on the observed temporal behavior of near surface wind velocity (Figure 1b). The primary goal behind this temporal geostrophic forcing is to simulate the strong decrease in friction velocity, $u_{*}$ in the nocturnal boundary layer as observed in HATS (Figure 2b).

[21] Figures $2 \mathrm{a}-2 \mathrm{c}$ shows the comparative evolution of near surface horizontal wind velocity, friction velocity and stability parameter, $\Delta_{h} / L$. The comparison between LES and HATS for the near-surface horizontal wind velocity is qualitatively good in terms of the temporal trend although the discrepancy seems to increase in the unstable part of the day. Since no estimates of geostrophic velocities are available from HATS campaign, a quantitative agreement between the simulation results and observations is not expected. Moreover, the observed differences in Figure 2a could possibly arise from the fact that the geostrophic wind forcing is assumed to be constant over height unlike some previous studies of the evolution of unstable convective boundary layer [Deardorff, 1974; Sun and Ogura, 1980]. As also remarked by Deardorff [1974, p. 104], "Variations in the Geostrophic wind must be modelled more accurately before satisfactory predictions of surface wind can be expected." The friction velocity $u_{*}$ shows a qualitatively similar decline in the nocturnal regime as compared to the HATS observations (Figure 2b). Recall that the surface heat flux boundary condition is a smoothed fit obtained from the surface heat flux observations (Figure 1a) and does not account for the stronger cooling fluxes observed during the evening transition, possibly causing the observed discrepancy between the LES results and observations of $u_{*}$ at the transition. It is 

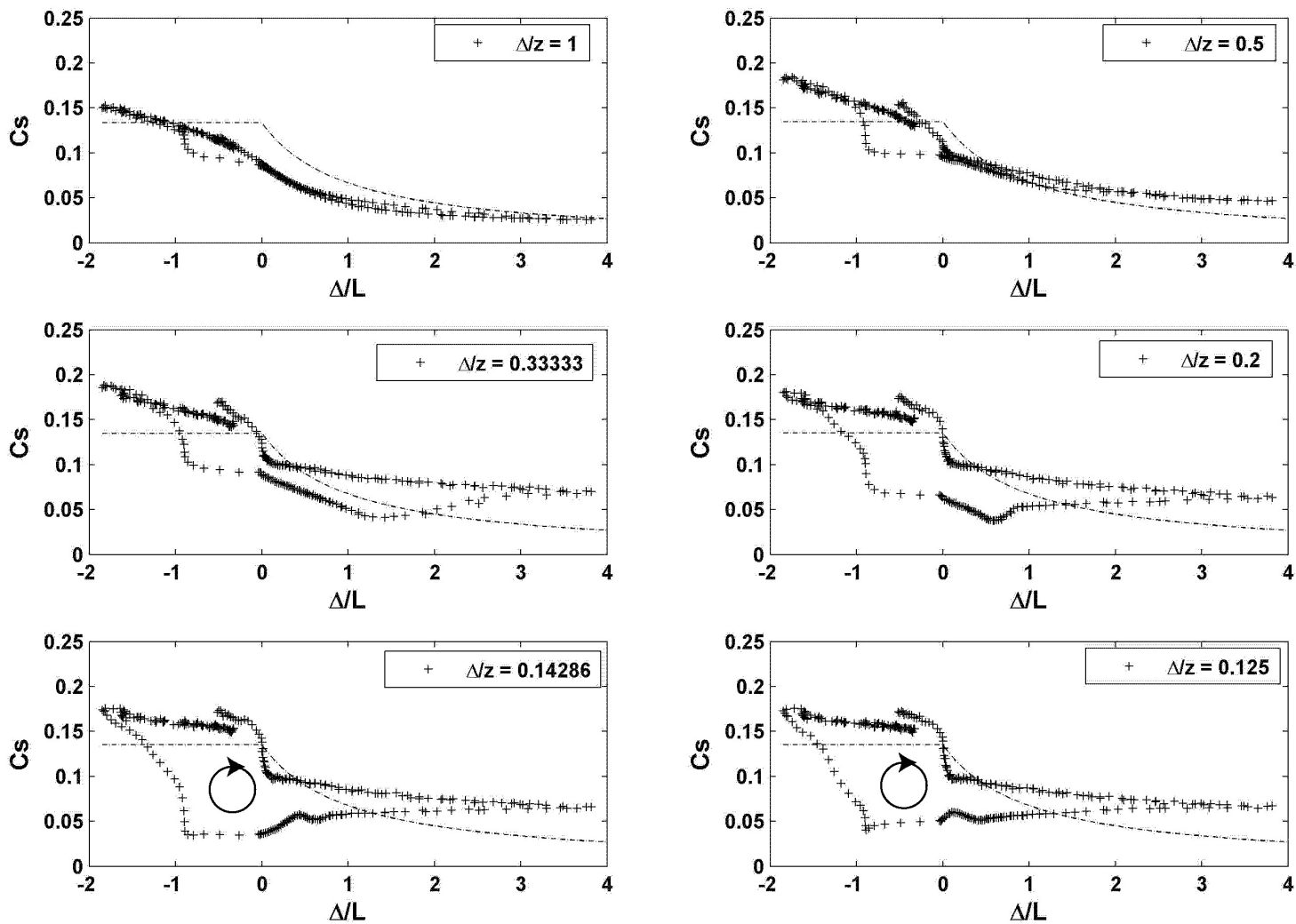

Figure 9. Smagorinsky coefficient, $C_{s}$, as a function of $\Delta / L$ for six vertical levels given by $\Delta / z=[1,0.5$, $0.33,0.2,0.142,0.125]$.
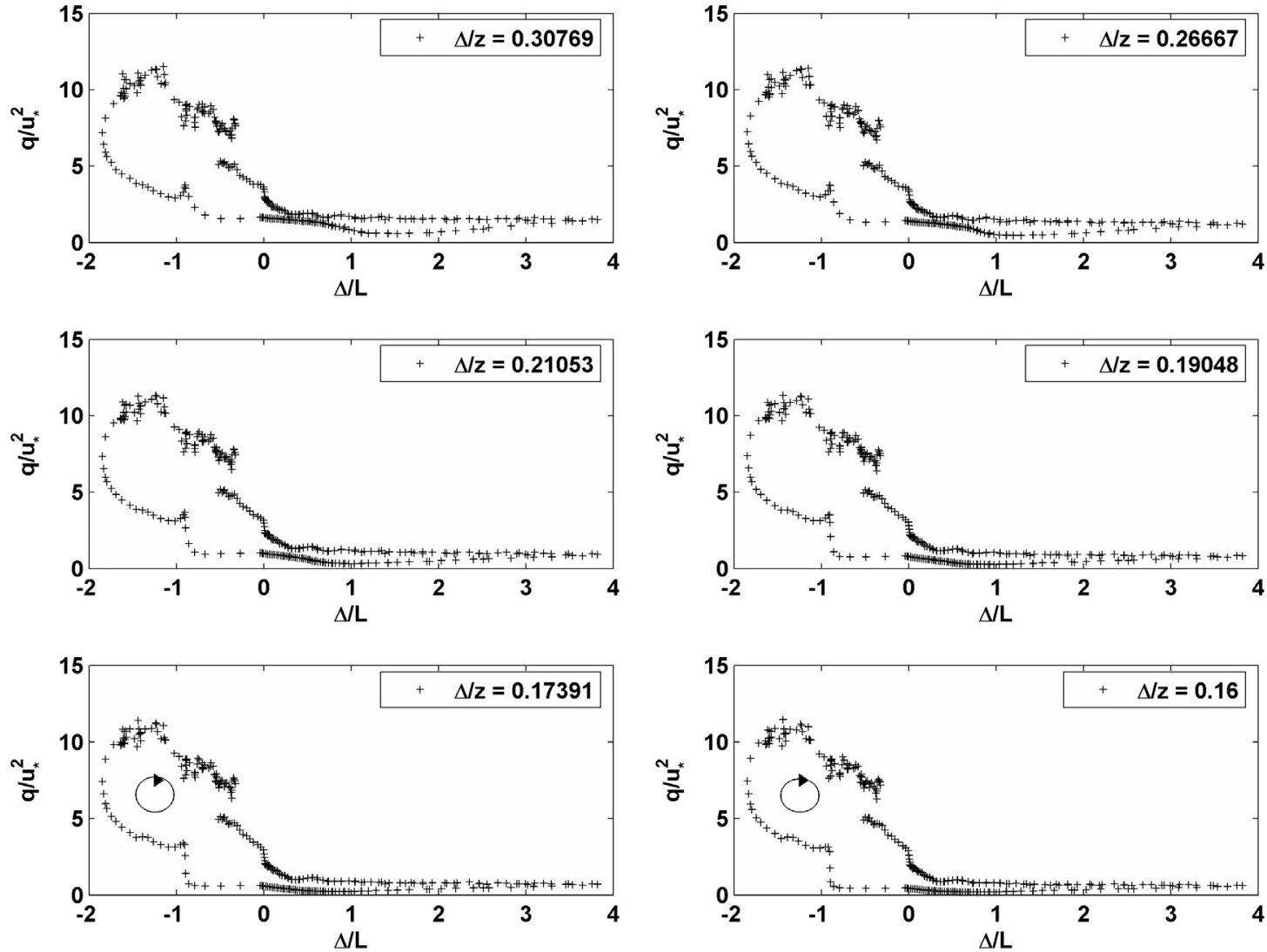

Figure 10. Nondimensional resolved turbulent kinetic energy, $q / u_{*}^{2}$, as a function of $\Delta / L$ for six vertical levels given by $\Delta / z=[0.30,0.26,0.21,0.19,0.17,0.16]$. 

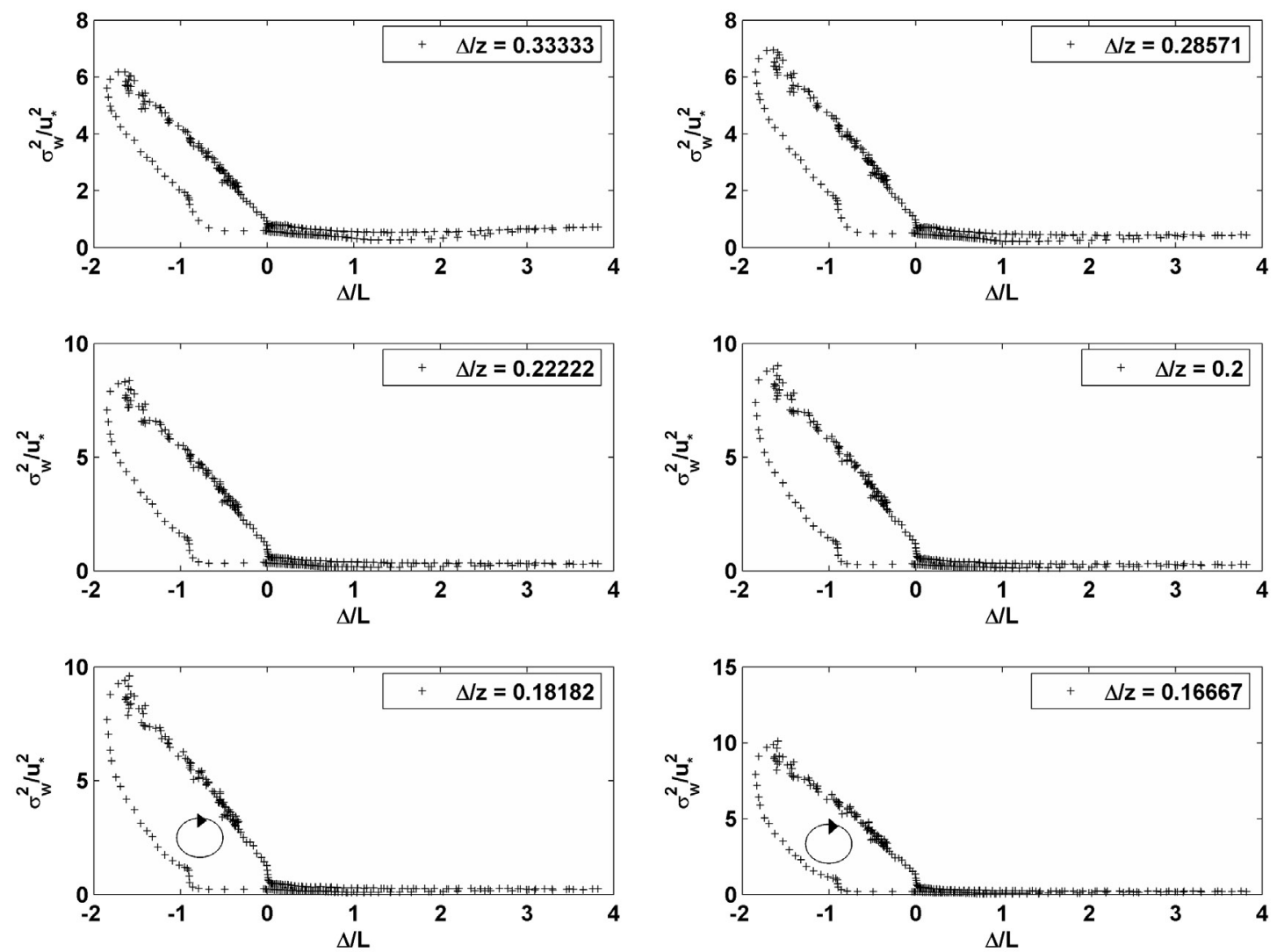

Figure 11. Nondimensional variance of vertical velocity, $\sigma_{w}^{2} / u_{*}^{2}$, as a function of $\Delta / L$ for six vertical levels given by $\Delta / z=[0.33,0.28,0.22,0.2,0.18,0.16]$.

encouraging that the LES produces the same trends as in the observations. Figure $2 \mathrm{c}$ shows the diurnal evolution of stability parameter $\Delta_{h} / L$. It represents the balance between the stability-governed characteristics of the simulated regime and the LES horizontal resolution $\Delta_{h}$. Note that $\Delta_{h}=\sqrt{\Delta_{x} \Delta_{y}}$ while $\Delta=\sqrt[3]{\Delta_{x} \Delta_{y} \Delta_{z}}$. The LES results are presented as a function of $\Delta_{h}$ in order to be consistent with the definition of filter size as $\Delta_{h}$ in HATS. For the sake of simplicity, we will henceforth use the symbol $\Delta$ in place of $\Delta_{h}$. The trend observed in $\Delta / L$ is consistent with the HATS observations except for the very strong excursions in the HATS data. We remark here that we have been able to simulate stable regimes up to $\Delta / L=4$. We also observed that at any fixed $\Delta$, there is a maximum strength of cooling which can be accurately simulated and any higher value of cooling (values leading to $\Delta / L>4$ ) would produce spurious oscillations and become numerically unstable. We believe that this behavior is a manifestation of the inability of eddy viscosity/eddy diffusivity SGS models to simulate strongly stable regimes. This observation, though not new [Saiki et al., 2000], still serves to accentuate the need for improvements in SGS models for stable boundary layers.

[22] We also plot the widely used stability $z_{i} / L$ parameter, where $z_{i}$ is the boundary layer height (Figure 2d). For the unstable part of the simulation, $z_{i}$ is based on location of the position of maximum downward heat flux, while $z_{i}$ for the stable regime is based on the location where the total stress has decayed to $5 \%$ of surface stress. $z_{i} / L$ attains large values up to 40 in the stable regime and it becomes -50 in the convective regime of the ABL.

\subsection{Vertical Structure of Atmospheric Turbulence}

[23] With the underlying surface being horizontally homogeneous, the main direction of variability is in the vertical, and so we now proceed to investigations of the influence of atmospheric stability on vertical structure of atmospheric turbulence.

[24] We start by looking at the daytime, convective portion of the ABL. Since the potential temperature $\widetilde{\theta}$ provides a good indication of the vertical structure of the CBL such as the surface layer and mixed layer, we begin by looking at its temporal evolution in Figure 3a. A gradual increase in $\widetilde{\theta}$ can be seen right after sunrise. The surface layer soon becomes convectively unstable with an increasing surface heat flux (Figure 4a). Vigorous turbulent mixing breaks out as is evident from the profile of momentum fluxes and resolved turbulent kinetic energy (Figures $4 \mathrm{~b}$ and $4 \mathrm{c}$ ). As the surface heat flux steadily increases, there is a rapid growth in the vertical extent of the mixed layer. With the CBL growing by entraining from the overlying inversion layer, the thickness of the turbulent layer increases over the course of the day with concomitant decrease in the vertical extent of the overlying inversion layer. It is also clear that the top of the turbulent layer coincides with the bottom of the overlying capping inversion layer in the CBL. The narrow white band above the convective boundary layer between the hours 09001600 in Figure 4a is a zone of maximum downward heat 

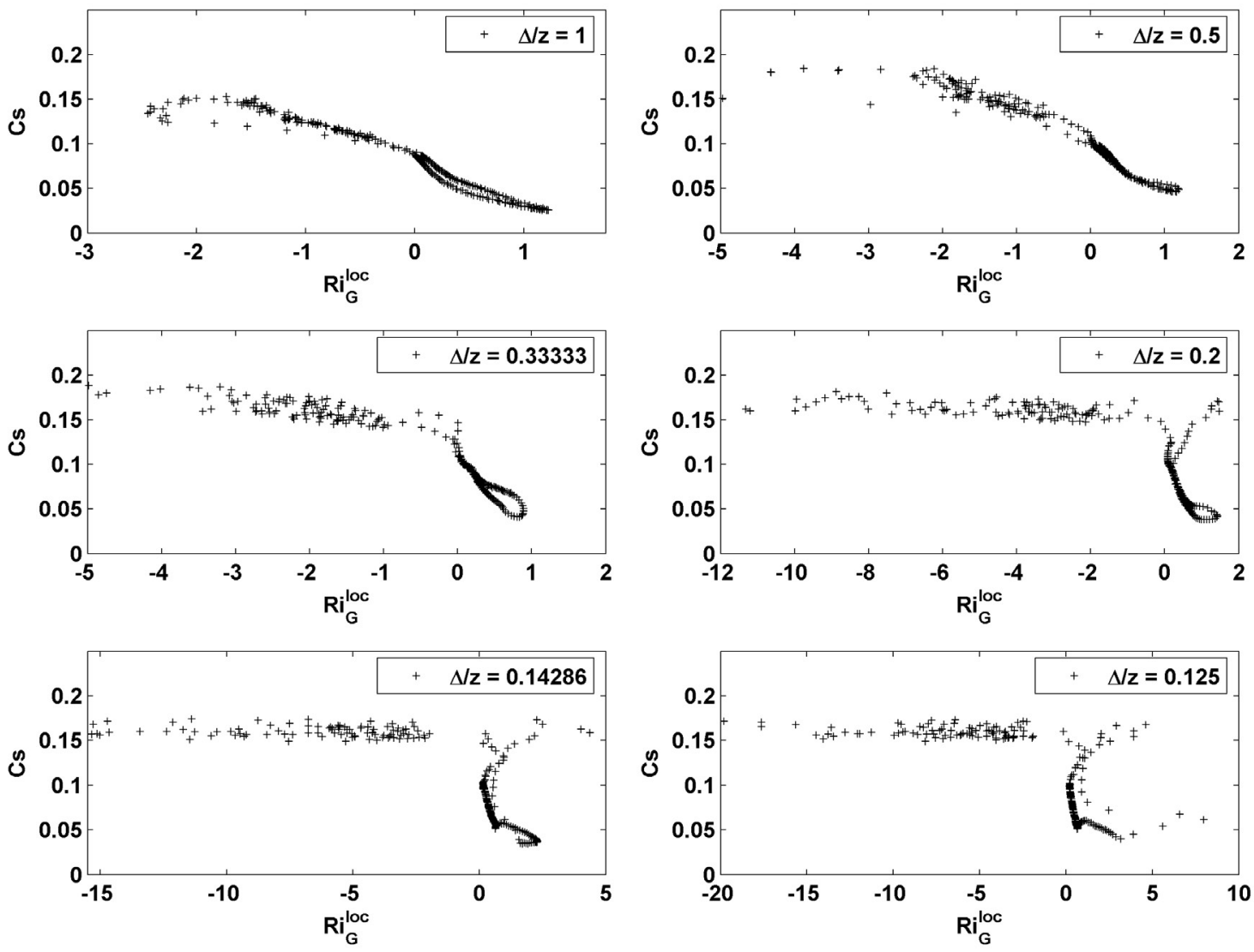

Figure 12. Smagorinsky coefficient, $C_{s}$, as a function of $R i_{g}^{l o c}$ for six vertical levels given by $\Delta / z=$ $[1,0.5,0.33,0.2,0.142,0.125]$.

flux. This zone owing to its well-established connection with the convective boundary layer top and the bottom of the entrainment zone [Willis and Deardorff, 1974], serves to show the increase in the vertical extent of the convective boundary layer (CBL) by entrainment. This is also manifested in an "overshoot" region characterized by strong vertical mean gradients of $\theta$ (due to penetrative convection) along with the progressive growth of the wellmixed CBL as can be seen in Figure 3c. These results are in good qualitative agreement with the modeling results of André et al. [1978] and field observations of Kaimal et al. [1976]. The velocity profile in the daytime convective boundary layer has the characteristic well-mixed profile with a logarithmic increase in surface layer and a constant value in the mixed layer as can be seen in Figure 5a.

[25] The nocturnal stable regime displays weaker winds with a low-level jet characterizing the presence of a strongly sheared region above the surface layer. The onset of nocturnal boundary layer dynamics is triggered by the rapid collapse of the convective boundary layer after sunset. The ensuing surface cooling leads to the creation of a surface inversion layer (Figures $3 \mathrm{a}$ and $3 \mathrm{~b}$ ). The stratified surface inversion layer serves to strongly attenuate the mechanical turbulence. The nearly vanishing turbulent stresses lead to the onset of a damped inertial oscillation which leads to the subsequent appearance of an inversion layer supergeostrophic wind also known as low-level jet (LLJ) [Blackadar, 1957; Van Ulden and Wieringa, 1996]. The evolution of the LLJ can be clearly seen in Figure $5 b$ where the LLJ has a broad maximum at 2300 hours which evolves into a welldefined narrow inversion maximum at 0300 hours. Notice in Figures $4 \mathrm{~b}$ and $4 \mathrm{c}$ that as the surface starts to cool after sunset (1800-1900 hours), the near-surface turbulence is suppressed by the growing surface inversion (Figure $3 \mathrm{~b}$ ) while turbulence persists in the residual layer. This "residual" turbulence, being decoupled from the surface, also aids in the formation of the low-level jet [Mahrt, 1999]. The temporal evolution of the stable boundary layer can be characterized with three important length scales: the depth of the turbulent layer $\left(h_{t}\right)$, depth of the surface inversion layer $\left(h_{i}\right)$ and height of the LLJ $\left(h_{J}\right) . h_{J}$ is defined as the vertical location of maximum wind speed, $h_{t}$ is calculated by first determining the vertical location where the momentum flux decays to $5 \%$ of its surface value and then, linearly interpolating to find the height where the fluxes vanish [Kosović and Curry, 2000] and $h_{i}$ is defined as the depth of the layer where $\partial\langle\theta\rangle / \partial z$ exceeds $3.5 \times 10^{-3} \mathrm{Km}^{-1}$ [André and Mahrt, 1982]. As can be seen in Figures $5 \mathrm{~b}$ and $6, h_{J}$ decreases to a minimum of $\sim 125 \mathrm{~m}$ at 0300 hours corresponding to a period of strong cooling and subsequently starts to increase as the strength of the cooling decreases. As the SBL evolves over the course of the night, $h_{t}$ decreases and $h_{i}$ increases as can also be seen in Figure 6 [André and Mahrt, 1982; Smedman, 1990]. This is caused by the interplay between the opposing forces of stratification and mechanical turbulence and can be better gauged by looking at the temporal evolution of momentum and heat fluxes in the nocturnal boundary layer in Figure 7 . The increasing strength of surface cooling causes a growing surface inversion layer 

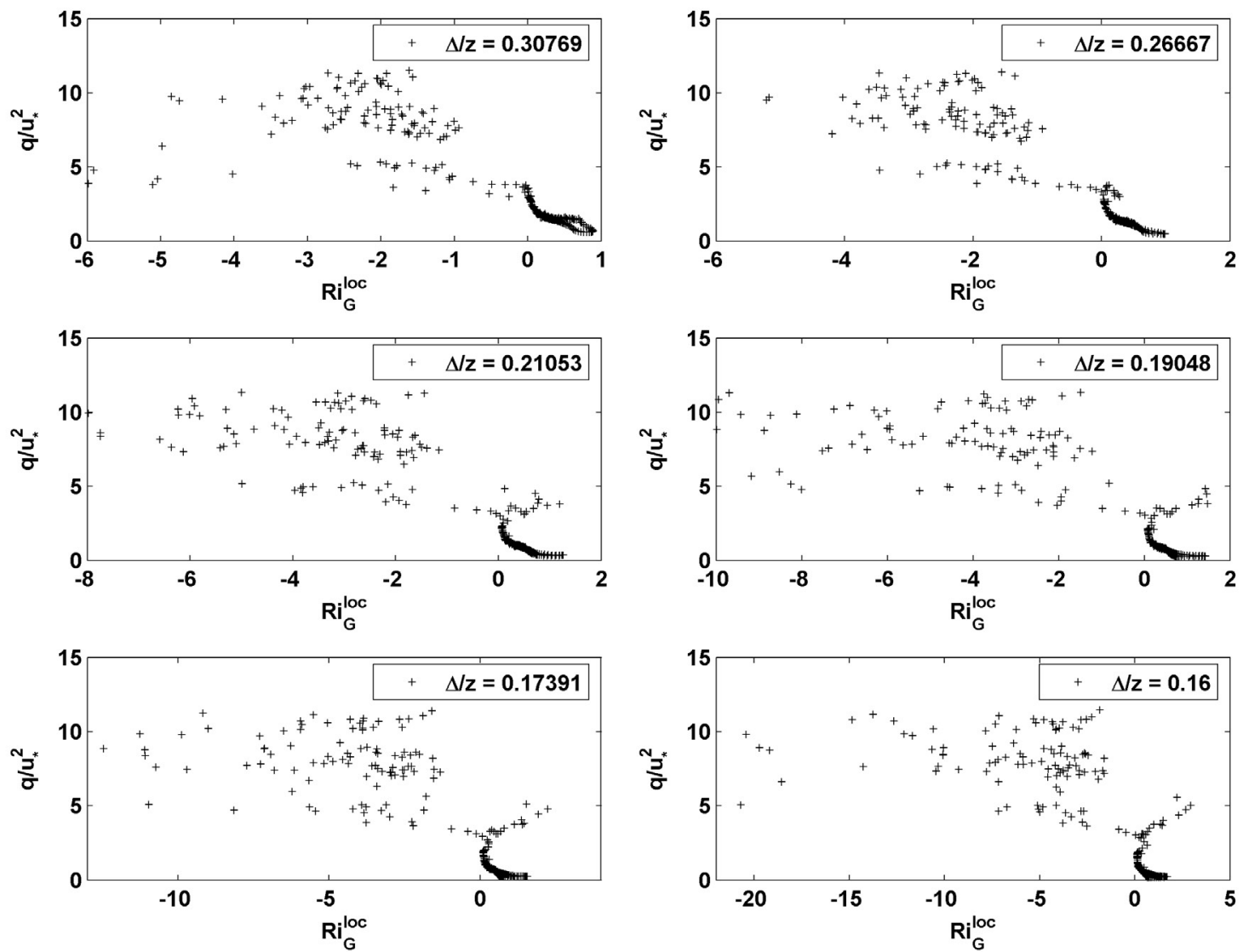

Figure 13. Nondimensional resolved turbulent kinetic energy, $q / u_{*}^{2}$, as a function of $R i_{g}^{l o c}$ for six vertical levels given by $\Delta / z=[0.30,0.26,0.21,0.19,0.17,0.16]$.

(Figure $3 b$ ) which thereby leads to increased suppression of turbulent mixing and hence the decay of the turbulent layer $\left(h_{t}\right)$.

[26] An additional observation worth mentioning relates to the behavior of SBL velocity profiles from field observations in the work by André et al. [1978]. The observational profiles show that the wind velocity decays with height above the LLJ with the decay rate being higher in the overlying inversion layer compared to the residual layer. The profiles obtained from RANS model runs of André et al. [1978] are not able to capture the characteristics of the SBL velocity profiles with the prime difference being the localized presence of the LLJ in the observations. In a qualitative sense, the SBL profiles obtained from the LES simulation and shown in Figure $5 \mathrm{~b}$ are remarkably similar to the field observations (day 33 of the Wangara data) of André et al. [1978].

\subsection{Evolution of Subgrid Parameter, $\boldsymbol{C}_{\boldsymbol{s}}$}

[27] Kleissl et al. [2004] showed that the Smagorinsky coefficient, in addition to being a function of space and time, is strongly affected by atmospheric stability and shear. Figure 8 shows the diurnal evolution of the vertical profile of Smagorinsky coefficient obtained from the present LES results. The tuning-free scale-dependent parameter obtained during the simulation from equation (5) reacts rapidly to the change in atmospheric stability. $C_{s}$ is higher in the mixed layer and decreases in the stable, nocturnal regime. The coefficient has smaller values both in the near-surface region and in the overlying inversion layer, albeit for different reasons, namely enhanced shear for the former and stratification-damped turbulence for the latter. While the observed $C_{s}$ profiles during daytime and nighttime are similar to the results obtained from the LES studies of quasi-steady unstable and stable boundary layers of Kleissl et al. [2006], the magnitude and vertical extent of $C_{s}$ is clearly a function of the atmospheric stability. In addition, the values of $C_{s}$ change rapidly around the morning and the evening transition regimes underscoring the benefits of a dynamic tuning-free procedure for determination of the parameter. Note that the impact of such a large variability in magnitude of $C_{S}$ as observed during the transition regimes can only be gauged using simulations of the diurnal cycle of the ABL.

[28] The observation that the change in the stability originating at the surface is conveyed rapidly through the entire structure of the ABL is not surprising and has been reported by several others in the past [Grant, 1997; Kaimal et al., 1976]. In addition, the dynamics of the ABL have been found to differ markedly between the morning and the evening transitions [Grimsdell and Angevine, 2002]. The next section probes this issue and its ramifications on boundary layer scaling in more detail.

\subsection{Stability Dependence and Scaling in the Diurnal Cycle}

[29] In order to better assess the behavior of computed variables as a function of atmospheric stability, we plot time 

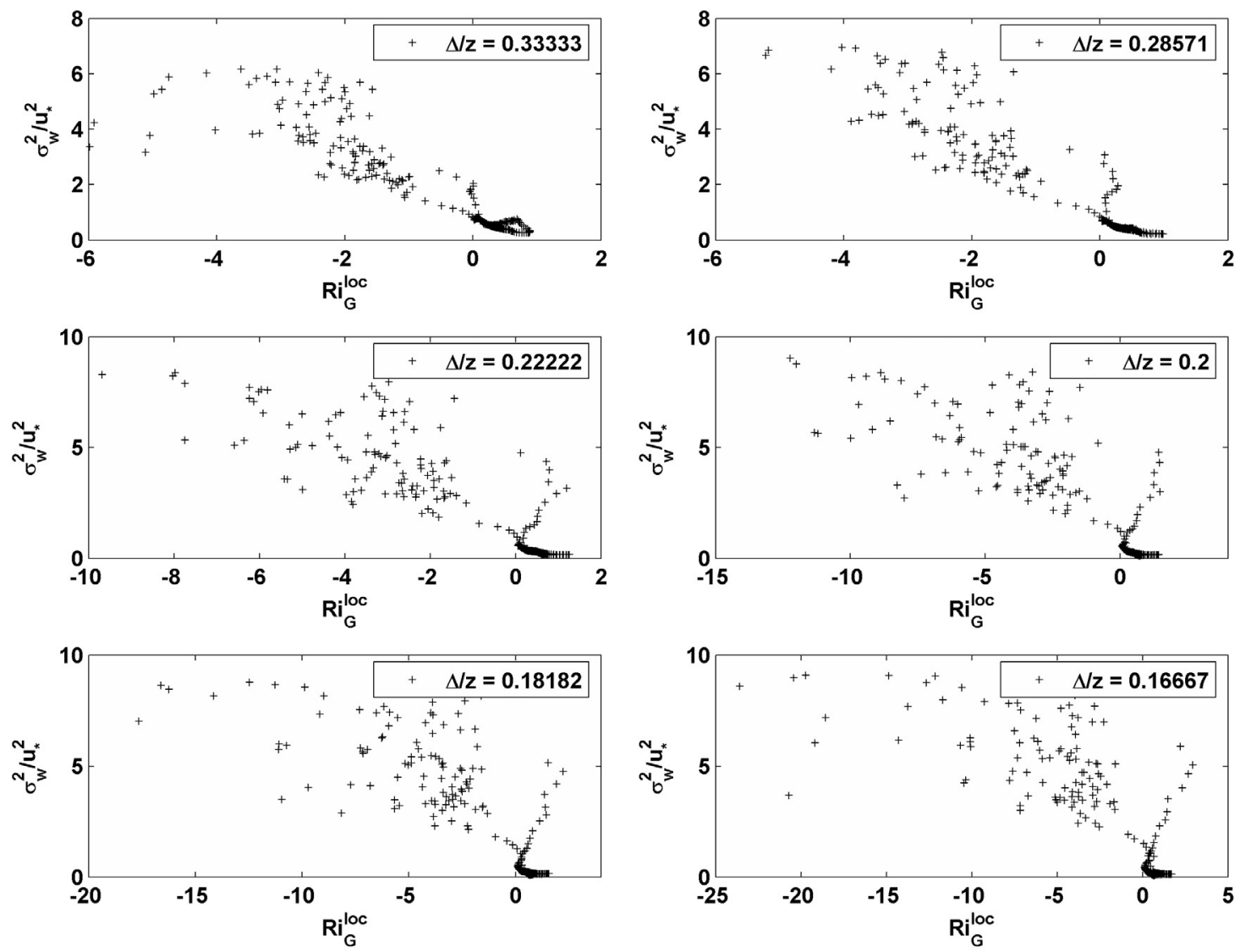

Figure 14. Nondimensional variance of vertical velocity, $\sigma_{w}^{2} / u_{*}^{2}$ as a function of $R i_{g}^{l o c}$ for six vertical levels given by $\Delta / z=[0.33,0.28,0.22,0.2,0.18,0.16]$.

series of plane-averaged variables as a function of $\Delta / L$ for the entire diurnal cycle at different vertical levels.

[30] We start by looking at the behavior of the planeaveraged $C_{s}$ as a function of $\Delta / L$ plotted for six vertical levels $(\Delta / z=[1,0.5,0.33,0.2,0.142,0.125])$ as shown in Figure 9. The empirical relation developed in Kleissl et al. [2004] relating $C_{s}$ to height and stability given by

$$
C_{s}=C_{0}\left[1+R\left(\frac{\Delta}{L}\right)\right]^{-1}\left[1+\left(\frac{C_{0}}{\kappa} \frac{\Delta}{z}\right)^{n}\right]^{-1 / n}
$$

where $R(x)=\mid \begin{array}{cc}x & \text { if } x \geq 0 \\ 0 & \text { otherwise }\end{array}, C_{0}=0.135$ and $n=3$ is also plotted for comparison. Note that since $C_{S}$ is plotted as a function of $\Delta / L$, the points belonging to similar stability regime are lumped together irrespective of their chronological occurrence. The trend concerning the behavior of $C_{s}$ as a function of $\Delta / L$ is in good agreement with the empirical relation given by equation 11 . Note however, that while the coefficient value seems to level off to a plateau for unstable conditions for higher vertical levels in agreement with Kleissl et al. [2004], this is not true for the near-surface levels where $C_{s}$ seems to gradually increase with decrease in $\Delta / L$ in $\mathrm{CBL}(\Delta / L<0)$.

[31] We also observe that the collapse of $C_{S}$ as a function of $\Delta / L$ becomes worse as we move away from the surface, as is evident from the increasing spread between the $C_{S}$ values with similar $\Delta / L$ values but differing chronological occurrence in the diurnal cycle. This "hysteresis"-like behavior occurs due to the difference between the morning and evening transition regimes of the diurnal cycle of the ABL. It is worth mentioning that this behavior is initially evident in the convective regime of the profile $(-1 \leq$ $\Delta / L \leq 0)$ and eventually spreads to the stable regimes as $\Delta / z$ decreases. In addition, the width of the hysteresis region also increases with increasing distance from the surface (decreasing values of $\Delta / z$ ). It is known that there is an underlying physical difference between the morning and evening transitions of the ABL, mainly owing to the difference between the adjustment timescales [Caughey, 1977; Kaimal et al., 1976]. Note, however, that there is no evidence of this behavior in the results from the HATS experiments, most likely due to the low measurement heights $(<10 \mathrm{~m})$. Consistent with this, note that in LES, there is almost no presence of hysteresis in the near-surface region $(\Delta / z \geq 1)$.

[32] We see similar behavior in profiles of resolved turbulent kinetic energy (TKE), $q$ (defined as $q=0.5\left[\left\langle\widetilde{u}^{\prime 2}\right\rangle+\left\langle\widetilde{v}^{2}\right\rangle\right.$ $\left.+\left\langle\widetilde{w}^{\prime 2}\right\rangle\right]$ ) nondimensionalized by $u_{*}^{2}$ as a function of $\Delta / L$ plotted for vertical levels of $\Delta / z=[0.30,0.26,0.21,0.19$, $0.17,0.16]$, and shown in Figure 10. The choice of different height levels for $C_{s}$ and $q$ is based on the observation that while $C_{s}$ is a variable scaling the SGS stress and acquires significance in the near-surface region due to decreasing length scales, $q$ is a sum of resolved velocity variances and is well defined in a scaling sense in regions where the resolved fraction of the flow is significantly larger than the SGS portion. This was raised 

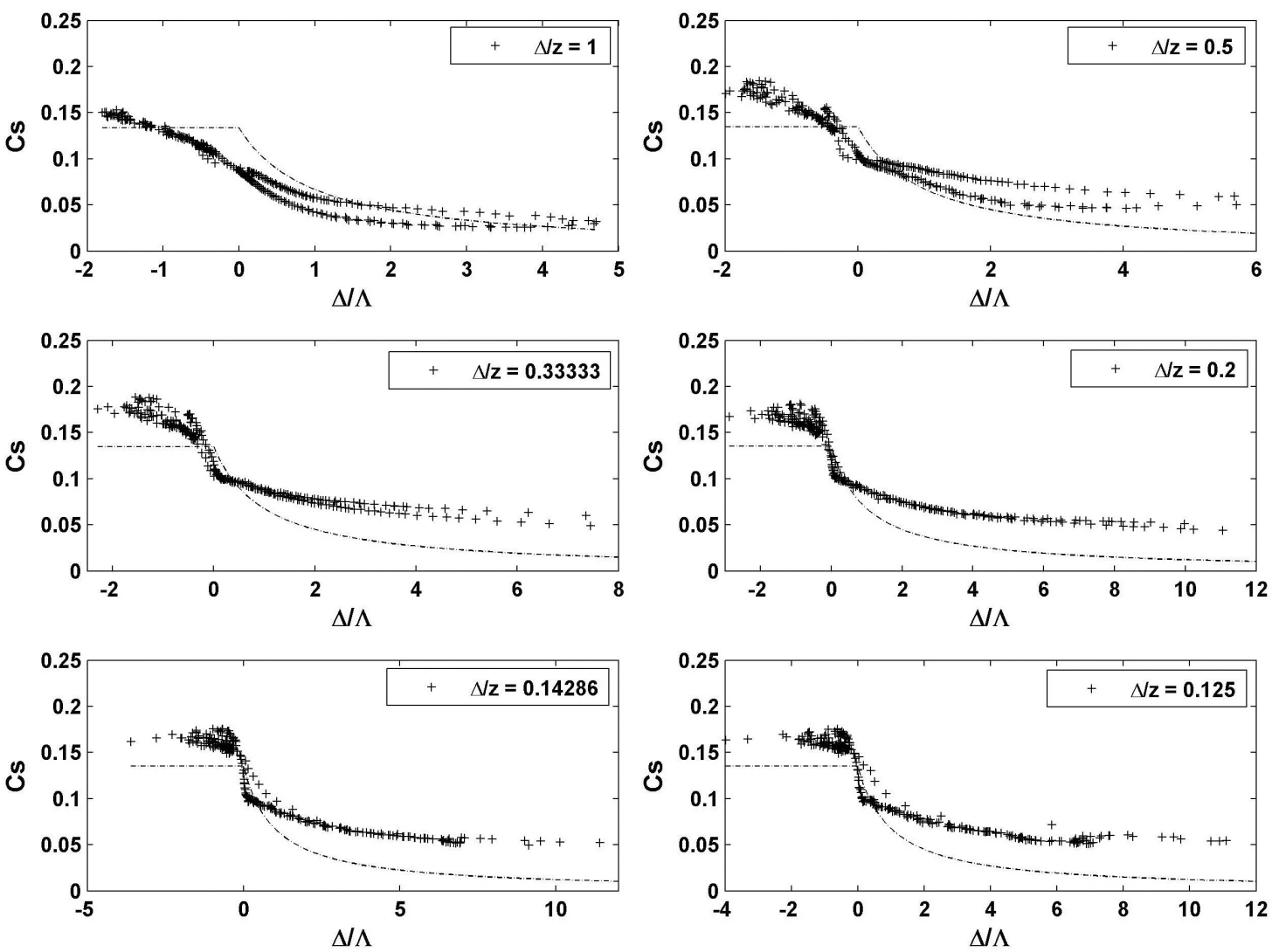

Figure 15. Smagorinsky coefficient, $C_{S}$, as a function of $\Delta / \Lambda$ for six vertical levels given by $\Delta / z=[1$, $0.5,0.33,0.2,0.142,0.125]$.

by Brown et al. [1994] where in the context of scaling resolved variances, points were chosen in the region above $0.12 z_{i}$. The presence of hysteresis is evident at all vertical levels in the case of $q$ with strong presence in the unstable and the transition regime and weak presence in the nearsurface stable regime. Note that the near constancy of $q$ in nearly the entire stable regime is more likely the consequence of very small turbulent stresses in the nocturnal boundary layer than the absence of hysteresis. A similar analysis performed on resolved vertical velocity variance $\sigma_{w}^{2}$ nondimensionalized by $u_{*}^{2}$ for $\Delta / z=[0.33$, $0.28,0.22,0.2,0.18,0.16]$ produces analogous results (Figure 11). Note however, that the stability range of the hysteresis region is smaller as compared to the range observed for $q$. The presence of hysteresis in the nearsurface regions $(\Delta / z \geq 0.2)$ is highly depleted in comparison to the observation for $q$, leading to the conclusion that the horizontal eddy kinetic energy, $e_{h}=$ $0.5\left(\left\langle\widetilde{u}^{2}\right\rangle+\left\langle\widetilde{v}^{2}\right\rangle\right)$ dominates over the vertical eddy kinetic energy, $e_{w}=0.5 \sigma_{w}^{2}$ in the stable regime. Specifically, a plot (not shown) of the ratio $e_{h} / e_{w}$ as a function of $\Delta / L$ for the six $\Delta / z$ levels reveals that $e_{h} / e_{w}$ is $\geq 3$ in the stable boundary layer. A similar deduction was also made by André et al. [1978] based on their RANS modeling results. At this point, it is clear that the observed hysteresis is shown in all three variables $\left(C_{s}, q, \sigma_{w}^{2}\right)$ presented in this section and therefore is a general issue affecting their scaling in the diurnal ABL.

[33] A strong possible source of hysteresis is the usage of a surface layer parameter $\Delta / L$ to scale variables outside the surface layer. A better strategy aimed at capturing the essence of the multistability characteristics of the ABL should encompass a combination of standard boundary layer scalings (e.g., $z, L, u_{*}, \Delta$ ) rather than comparing them individually [Holtslag and Nieuwstadt, 1986]. Therefore we now evaluate other possible scaling parameters in an attempt to find an appropriate choice to scale the profiles of $C_{s}, q$ and $\sigma_{w}^{2}$ across multistability regimes and distance from the surface.

[34] One possibility is the local gradient Richardson number, $R i_{g}^{l o c}$ given by

$$
R i_{g}^{l o c}=\frac{g}{\langle\widetilde{\theta}\rangle} \frac{\frac{\partial\langle\widetilde{\theta}\rangle}{\partial z}}{\frac{\partial\langle\widetilde{U}\rangle^{2}}{\partial z}+\frac{\partial\langle\widetilde{V}\rangle^{2}}{\partial z}},
$$

where the superscript loc refers to the use of variables from the same vertical level. The averaging $(\langle\rangle)$ is performed both over horizontal planes and in time. Note that $R i_{g}^{l o c}$ is a parameter varying in the vertical as compared to the constant $\Delta / L$ since it is based on local parameters unlike the latter. Figure 12 shows the profile of $C_{s}$ as a function of $R i_{g}^{l o c}$. The hysteresis effect is visibly reduced for the higher vertical levels. However, this reduction of hysteresis is accompanied by increased scatter with decreasing $\Delta / z$ and a spurious behavior of the profile in the stable region. The increased scatter is caused by the near-constant profiles of mean horizontal velocity and mean potential temperature 

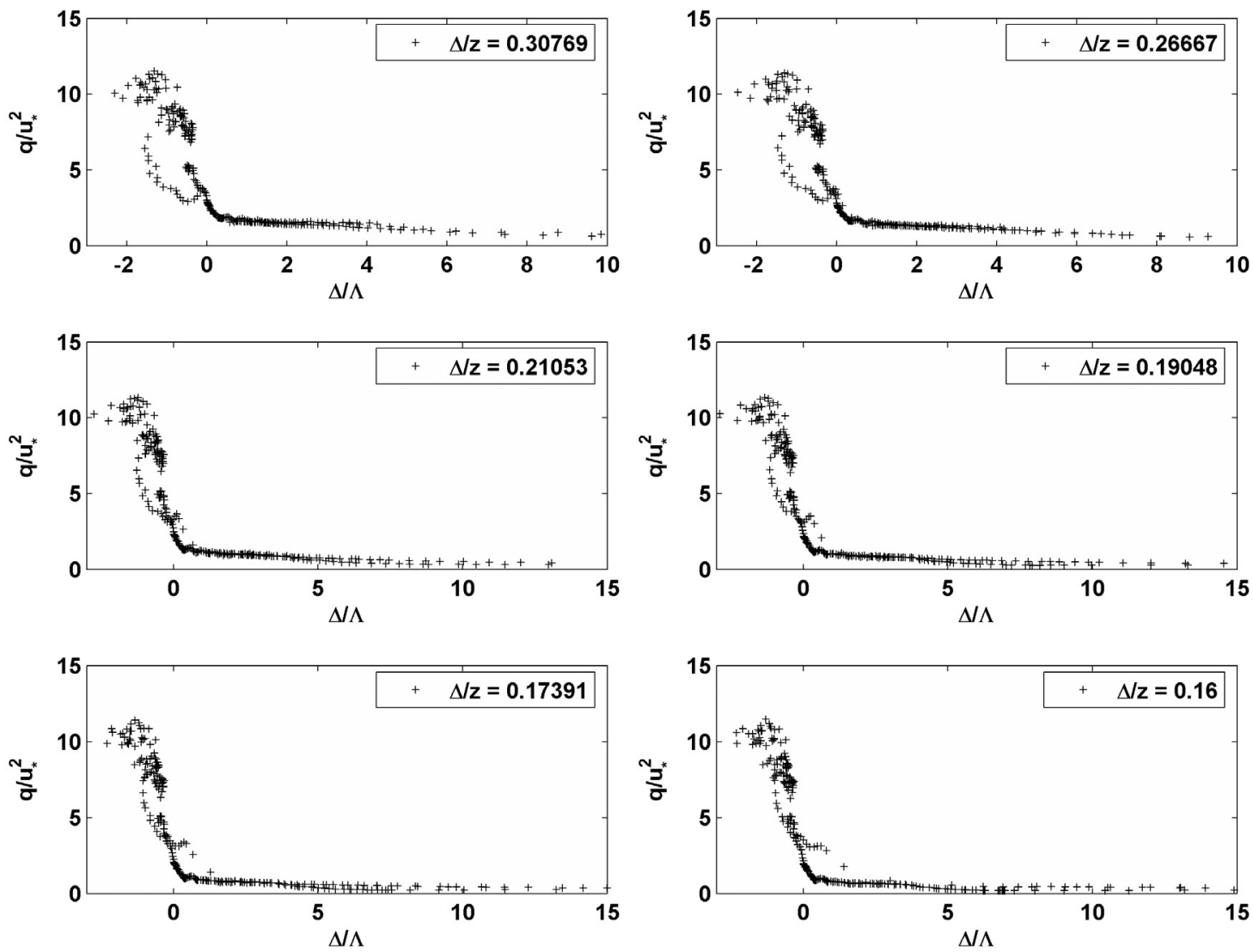

Figure 16. Nondimensional resolved turbulent kinetic energy, $q / u_{*}^{2}$, as a function of $\Delta / \Lambda$ for six vertical levels given by $\Delta / z=[0.30,0.26,0.21,0.19,0.17,0.16]$.

in the mixed layer during the convective regime. The resulting vertical gradients are extremely small in magnitude resulting in strong fluctuations of $R i_{g}^{l o c}$ in the mixed layer.

[35] The performance of $R i_{g}^{l o c}$ as a scaling parameter is further tested by plotting $q / u_{*}^{2}$ and $\sigma_{w}^{2} / u_{*}^{2}$ as a function of $R i_{g}^{l o c}$ as shown in Figures 13 and 14, respectively. The profiles of $q / u_{*}^{2}$ and $\sigma_{w}^{2} / u_{*}^{2}$ are affected by significant scatter in the unstable regimes. The same inference also holds for smaller values of $\Delta / z$ in stable conditions. The increased scatter obscures the impact of $R l_{g}^{l o c}$ scaling on hysteresis. The scatter increases significantly when $\Delta / z \leq 0.2$ and the performance with respect to hysteresis becomes worse in comparison to results obtained with the scaling by $\Delta / L$ (see corresponding plots in Figure 10). On the basis of these observations, one cannot convincingly argue that $R i_{g}^{\text {loc }}$ is the universal scaling parameter for all stability regimes, despite improvements observed with respect to near-surface hysteresis.

[36] Next, we consider the local scaling hypothesis of Nieuwstadt [1984] which has been found to be applicable to flows in stable boundary layers [Derbyshire, 1990; Mahrt, 1999]. According to this hypothesis, the nondimensional variables can be described as a function of $\Delta / \Lambda$ where

$$
\Lambda=-\frac{\tau_{l o c}^{3 / 2}}{k \frac{g}{\langle\widetilde{\theta}\rangle} \overline{w^{\prime} \theta^{\prime}}} ; \tau_{l o c}=\sqrt{\left\langle u^{\prime} w^{\prime}\right\rangle^{2}+\left\langle v^{\prime} w^{\prime}\right\rangle^{2}} .
$$

We try to extend the concept of local scaling to the diurnal cycle of ABL. Figure 15 shows the results obtained with the implementation of local scaling for the profiles of $C_{s}$. The local scaling eliminates the hysteretic behavior almost entirely and has a compact range for $\Delta / \Lambda$, unlike $R i_{g}^{l o c}$. The hysteresis region has collapsed yielding a profile similar to the empirical profile from HATS using equation (11) with $L$ replaced by $\Lambda$ :

$$
C_{s}=C_{0}\left[1+R\left(\frac{\Delta}{\Lambda}\right)\right]^{-1}\left[1+\left(\frac{C_{0}}{\kappa} \frac{\Delta}{z}\right)^{n}\right]^{-1 / n} .
$$

Note that the LASD SGS model yields coefficient values that systematically exceed those given by equation (14) from HATS. In addition, one can clearly see in Figure 15 that the shape of the curve and the collapse are similar at all plotted values of $\Delta / z$.

[37] The result for $q / u_{*}^{2}$ can be seen in Figure 16 where local scaling again achieves a better collapse i.e., less hysteresis. Notice that while some remnants of hysteresis are seen in the unstable regime for $\Delta / z=[0.3,0.26]$, the hysteresis present at the same locations in the stable regime based upon $\Delta / L$ scaling (Figure 10) has been mostly eliminated. The performance of $\Delta / \Lambda$ as a scaling parameter becomes increasingly better with decreasing $\Delta / z$. A similar performance is observed for the case of $\sigma_{w}^{2} / u_{*}^{2}$ plotted as a function of $\Delta / L$ as shown in Figure 17, except for the 

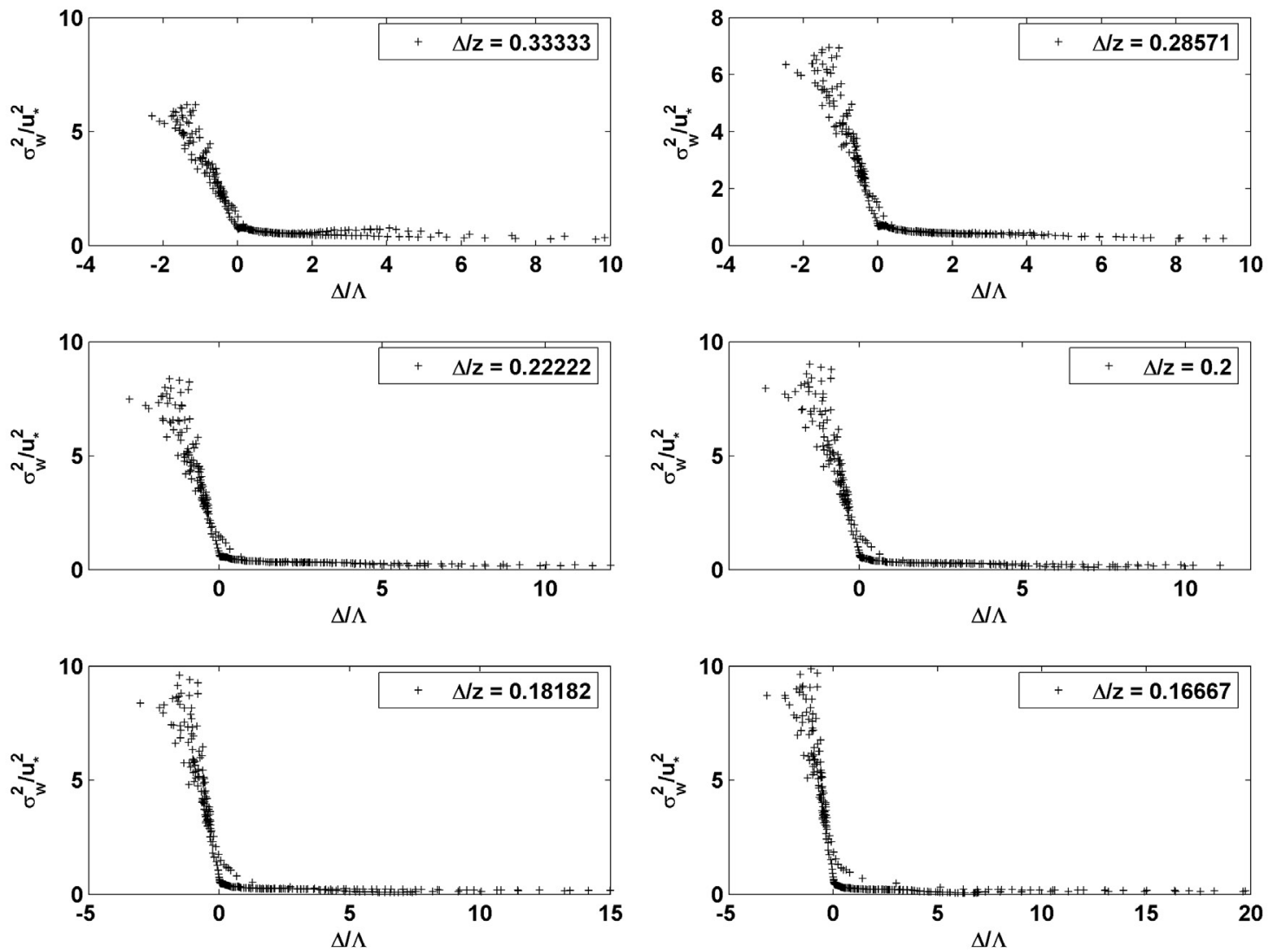

Figure 17. Nondimensional variance of vertical velocity, $\sigma_{w}^{2} / u_{*}^{2}$, as a function of $\Delta / \Lambda$ for six vertical levels given by $\Delta / z=[0.33,0.28,0.22,0.2,0.18,0.16]$.

observed increase in scatter in the unstable regime with decreasing $\Delta / z$.

[38] To summarize, while the performance of all three parameters, i.e., $\Delta / L, R i_{g}^{l o c}$, and $\Delta / \Lambda$, is comparable in the near-surface region $(\Delta / \Lambda \rightarrow \Delta / L$ as $z \rightarrow 0), \Delta / \Lambda$ performs better in achieving reduced hysteresis even with increasing distance from the surface. In addition, $\Delta / \Lambda$ performs remarkably well in the difficult diurnal transition regimes for all the three variables discussed above.

\section{Conclusions}

[39] A LES of the diurnal cycle of ABL forced by field observations from the HATS field experiment has been performed using the Lagrangian dynamic scale-dependent (LASD) SGS model. The results show the presence of characteristic features of the diurnal ABL such as entrainment-based growth of the CBL and evolution and dynamics of the nocturnal jet. The height and vertical extent of the nocturnal jet evolve continuously throughout the night with the jet moving closer to the surface with stronger surface cooling. The observed characteristics in LES results such as the mean velocity profiles were found to be qualitatively similar to previous experimental and modeling results [e.g., André et al., 1978; Kaimal et al., 1976]. The success of this simulation is in large part due to the autocalibration of the Smagorinsky coefficient from the resolved scales by the LASD model, leading to accurate simulation of the transition periods from day to night and vice versa. For instance, the simulation recovered from a stably stratified state with near-vanishing turbulence to evolve into a fully turbulent CBL after sunrise with concomitant adaptation of $C_{s}$. However, the eddy-viscosity formulations were found to become numerically unstable for more strongly stable regimes (with $\Delta / L>4$ ) at moderate resolution owing to spurious oscillations.

[40] We have shown that the profiles of $C_{s}, q / u_{*}^{2}$ and $\sigma_{w}^{2} / u_{*}^{2}$ plotted as a function of the stability parameter, $\Delta / L$ display a hysteretic behavior. The choice of $R i_{g}^{l o c}$ as the scaling parameter significantly reduces the hysteresis in the near-surface region but leads to increased scatter with increasing height. The application of Nieuwstadt's local scaling hypothesis by using $\Delta / \Lambda$ as the scaling parameter has been found to achieve very good collapse and near elimination of the hysteretic behavior for $C_{s}, q / u_{*}^{2}$, and $\sigma_{w}^{2} / u_{*}^{2}$. Our results thus show that the local scaling, despite being developed originally for the stable boundary layer, is appropriate to describe the scaling during the transition and convective regimes. In addition, the $C_{s}$ obtained from the dynamic model is described well by the empirical fit from HATS, modified using the parameter $\Delta / \Lambda$.

[41] Finally, we hope that LES simulations of the diurnal cycle of the ABL with improved accuracy stemming from the use of tuning-free dynamic SGS parameterization will provide accurate data that may serve in developing the next generation of turbulence closure models. Such models will 
inevitably be required for simulating hydrologic processes over very long, interannual timescales.

[42] Acknowledgments. This study was funded by the National Science Foundation (NSF) under NSF-WCR-0233646. Computations were made possible with the supercomputing grant from NCAR Supercomputing Division. We would like to acknowledge the valuable help from Stuart Chester in the process of rewriting the modular, parallel version of the LES code.

\section{References}

Albertson, J. D., and M. B. Parlange (1999), Surface length-scales and shear stress: Implications for land-atmosphere interaction over complex terrain, Water Resour. Res., 35, 2121-2132.

Albertson, J. D., and M. B. Parlange (2000), Natural integration of scalar fluxes from complex terrain, Adv. Water Resour., 23, 239-252.

André, J. C., and L. Mahrt (1982), The nocturnal surface inversion and influence on clear-air radiative cooling, J. Atmos. Sci., 39(4), 864-878.

André, J. C., G. de Moor, P. Lacarrére, G. Therry, and R. du Vachat (1978), Modeling the 24-hour evolution of the mean and turbulent structures of the planetary boundary layer, J. Atmos. Sci., 35, 1861-1883.

Andrén, A., A. R. Brown, J. Graf, P. J. Mason, C.-H. Moeng, F. T. M. Nieuwstadt, and U. Schumann (1994), Large-eddy simulation of a neutrally stratified boundary layer: A comparison of four computer codes, Q. J. R. Meteorol. Soc., 120, 1457-1484.

Antonopoulos-Domis, M. (1981), Large-eddy simulation of a passive scalar in isotropic turbulence, J. Fluid Mech., 104, 55-79.

Beare, R. J., and M. K. MacVean (2004), Resolution sensitivity and scaling of large-eddy simulations of the stable boundary layer, Boundary Layer Meteorol., 112, 257-281.

Blackadar, A. K. (1957), Boundary layer wind maxima and their significance for the growth of nocturnal inversions, Bull. Am. Meteorol. Soc., 38, 283-290.

Bou-Zeid, E., C. Meneveau, and M. B. Parlange (2004), Large-eddy simulation of neutral atmospheric boundary layer flow over heterogeneous surfaces: Blending height and effective surface roughness, Water Resour. Res., 40, W02505, doi:10.1029/2003WR002475.

Bou-Zeid, E., C. Meneveau, and M. B. Parlange (2005), A scale-dependent Lagrangian dynamic model for large eddy simulation of complex turbulent flows, Phys. Fluids, 17(2), 025105 .

Brown, A. R., P. J. Mason, and S. H. Derbyshire (1994), Large-eddy simulation of stable atmospheric boundary layers with a revised stochastic subgrid model, Q. J. R. Meteorol. Soc., 120, 1485-1512.

Brown, A. R., et al. (2002), Large-eddy simulation of the diurnal cycle of shallow cumulus convection over land, Q. J. R. Meteorol. Soc., 128(582), 1075-1093.

Brutsaert, W. (1982), Evaporation Into the Atmosphere: Theory, History and Applications, 299 pp., Springer, New York.

Businger, J. A. (1982), Equations and concepts, in Atmospheric Turbulence and Air Pollution Modelling, edited by F. T. M. Nieuwstadt and H. van Dop, pp. 1-33, Springer, New York.

Canuto, C., M. Y. Hussaini, A. Quarteroni, and T. A. Zang (1988), Spectral Methods in Fluid Dynamics, Springer, New York.

Caughey, S. J. (1977), Boundary layer turbulence spectra in stable conditions, Boundary Layer Meteorol., 11, 3-14.

Cheng, Y., and W. Brutsaert (2005), Flux-profile relationships for wind speed and temperature in the stable atmospheric boundary layer, Boundary Layer Meteorol., 114, 519-538.

Chlond, A., F. Muller, and I. Sednev (2004), Numerical simulation of the diurnal cycle of marine stratocumulus during FIRE-An LES and SCM modelling study, Q. J. R. Meteorol. Soc., 130(604), 3297-3321.

Deardorff, J. W. (1972), Numerical investigation of neutral and unstable planetary boundary layers, J. Atmos. Sci., 29, 91-115.

Deardorff, J. W. (1974), Three-dimensional numerical study of the height and mean structure of a heated planetary boundary layer, Boundary Layer Meteorol., 7, 81-106.

Derbyshire, S. H. (1990), Nieuwstadt's stable boundary layer revisited, Q. J. R. Meteorol. Soc., 116, 127-158.

Derbyshire, S. H. (1999), Stable boundary-layer modelling: Established approaches and beyond, Boundary Layer Meteorol., 90, 423-446.

Duynkerke, P. G., et al. (2004), Observations and numerical simulations of the diurnal cycle of the EUROCS stratocumulus case, Q.J. R. Meteorol. Soc., 130(604), 3269-3296.

Germano, M., U. Piomelli, P. Moin, and W. H. Cabot (1991), A dynamic subgrid-scale eddy viscosity model, Phys. Fluids A, 3, 1760-1765.
Grant, A. L. M. (1997), An observational study of the evening transition boundary layer, Q. J. R. Meteorol. Soc., 123, 657-677.

Grimsdell, A. W., and W. M. Angevine (2002), Observations of the afternoon transition of the convective boundary layer, J. Appl. Meteorol., 41(1), 3-11.

Hicks, B. B. (1976), Wind profile relationships from the Wangara experiments, Q. J. R. Meteorol. Soc., 102, 535-551.

Hogstrom, U. (1987), Non-dimensional wind and temperature profiles in the atmospheric surface layer: A re-evaluation, Boundary Layer Meteorol., $42,55-78$.

Holtslag, A. A. M., and H. A. R. De Bruin (1988), Applied modeling of the nighttime surface energy balance over land, J. Appl. Meteorol., 27, 689704.

Holtslag, A. A. M., and F. T. M. Nieuwstadt (1986), Scaling the atmospheric boundary layer, Boundary Layer Meteorol., 36, 201-209.

Horst, T. W., J. Kleissl, D. H. Lenschow, C. Meneveau, C. H. Moeng, M. B. Parlange, P. P. Sullivan, and J. C. Weil (2004), HATS: Field observations to obtain filtered fields from crosswind arrays of sonic anemometers in the atmospheric surface layer, J. Atmos. Sci., 61, $1566-1581$

Kaimal, J. C., J. C. Wyngaard, D. A. Haugen, O. R. Coté, Y. Izumi, S. J. Caughey, and C. J. Readings (1976), Turbulence structure in the convective boundary layer, J. Atmos. Sci., 33, 2152-2169.

Kang, H. S., and C. Meneveau (2002), Universality of large eddy simulation model parameters across a turbulent wake behind a heated cylinder, J. Turbulence, 3, pap. 32, doi:10.1088/1468-5248/3/1/032.

Khanna, S., and J. G. Brasseur (1997), Analysis of Monin-Obukhov similarity from large-eddy simulation, J. Fluid Mech., 345, 251-286.

Kleissl, J., C. Meneveau, and M. B. Parlange (2003), On the magnitude and variability of subgrid-scale eddy-diffusion coefficients in the atmospheric surface layer, J. Atmos. Sci., 60, 2372-2388.

Kleissl, J., M. B. Parlange, and C. Meneveau (2004), Field experimental study of dynamic Smagorinsky models in the atmospheric surface layer, J. Atmos. Sci., 61, 2296-2307.

Kleissl, J., V. Kumar, C. Meneveau, and M. B. Parlange (2006), Numerical study of dynamic Smagorinsky models in large-eddy simulation of the atmospheric boundary layer: Validation in stable and unstable conditions, Water Resour. Res., doi:10.1029/2005WR004685, in press.

Kosović, B., and J. A. Curry (2000), A large eddy simulation study of a quasi-steady, stably stratified atmospheric boundary layer, J. Atmos. Sci., 57, 1057-1068

Landau, L. D., and E. M. Lifshitz (1959), Fluid Mechanics, Course Theor. Phys., vol. 6, 532 pp., Addison-Wesley, Boston, Mass.

Lesieur, M., and O. Métais (1996), New trends in large-eddy simulations of turbulence, Annu. Rev. Fluid Mech., 28, 45-82.

Lilly, D. K. (1967), The representation of small-scale turbulence in numerical simulation experiments, in Proceedings of IBM Scientific Computing Symposium on Environmental Sciences, Yorktown Heights, NY, pp. 195210, IBM Data Process. Div., White Plains, N. Y.

Mahrt, L. (1999), Stratified atmospheric boundary layers, Boundary Layer Meteorol., 90, 375-396.

Mason, P. J. (1989), Large-eddy simulation of the convective atmospheric boundary layer, J. Atmos. Sci., 46, 1492-1516.

Mason, P. J., and S. H. Derbyshire (1990), Large eddy simulation of the stably-stratified atmospheric boundary layer, Boundary Layer Meteorol., $53,117-162$.

Meneveau, C., T. Lund, and W. Cabot (1996), A Lagrangian dynamic subgrid-scale model of turbulence, J. Fluid Mech., 319, 353-385.

Moeng, C.-H. (1984), A large-eddy simulation model for the study of planetary boundary-layer turbulence, J. Atmos. Sci., 41, 2052-2062.

Nieuwstadt, F. T. M. (1984), Some aspects of the turbulent stable boundarylayer, Boundary Layer Meteorol., 30, 31-55.

Nieuwstadt, F. T. M., and R. A. Brost (1986), The decay of convective turbulence, J. Atmos. Sci., 43, 532-546.

Nieuwstadt, F. T. M., P. J. Mason, C.-H. Moeng, and U. Schumann (1991), Large-eddy simulation of the convective boundary layer: A comparison of four computer codes, Turbulent Shear Flows, 8, 343-367.

Orszag, S. (1970), Transform method for calculation of vector coupled sums: Application to the spectral form of the vorticity equation, J. Atmos. Sci., 27, 890-895.

Orszag, S., and Y. Pao (1974), Numerical computation of turbulent shear flows, Adv. Geophys., 18A, 224-236.

Pope, S. B. (2000), Turbulent Flows, Cambridge Univ. Press, New York.

Porté-Agel, F. (2004), A scale-dependent dynamic model for scalar transport in large-eddy simulations of the atmospheric boundary layer, Boundary Layer Meteorol., 112, 81-105. 
Porté-Agel, F., C. Meneveau, and M. B. Parlange (2000a), A scale-dependent dynamic model for large-eddy simulation: Application to a neutral atmospheric boundary layer, J. Fluid Mech., 415, 261-284.

Porté-Agel, F., M. B. Parlange, C. Meneveau, W. Eichinger, and M. Pahlow (2000b), Subgrid-scale dissipation in the atmospheric surface layer: Effects of stability and filter dimension, J. Hydrometeorol., 1, 75-87.

Porté-Agel, F., M. Pahlow, C. Meneveau, and M. Parlange (2001), Atmospheric stability effect on subgrid-scale physics of large-eddy simulation, Adv. Water Resour., 24, 1085-1102.

Povitsky, A., and P. J. Morris (2000), A higher-order compact method in space and time based on parallel implementation of the Thomas algorithm, J. Comput. Phys., 161(1), 182-203.

Saiki, E., C.-H. Moeng, and P. Sullivan (2000), Large-eddy simulation of the stably stratified planetary boundary layer, Boundary Layer Meteorol., 95, $1-30$.

Schmidt, H., and U. Schumann (1989), Coherent structure of the convective boundary layer derived from large-eddy simulations, J. Fluid Mech., 200, $511-562$.

Smagorinsky, J. (1963), General circulation experiments with the primitive equations. I. The basic experiment, Mon. Weather Rev., 91, 99-164.

Smedman, A.-S. (1990), Some turbulence characteristics in the stable atmospheric boundary layer, J. Atmos. Sci., 48, 856-868.

Sorbjan, Z. (1997), Decay of convective turbulence revisited, Boundary Layer Meteorol., 82, 501-515.

Stoll, R., and F. Porté-Agel (2006), Dynamic subgrid-scale models for momentum and scalar fluxes in large-eddy simulations of neutrally stratified atmospheric boundary layers over heterogeneous terrain, Water Resour. Res., 42, W01409, doi:10.1029/2005WR003989.

Stull, R. B. (1997), An Introduction to Boundary Layer Meteorology, Springer, New York.

Sun, W.-Y., and Y. Ogura (1980), Modeling the evolution of the convective boundary layer, J. Atmos. Sci., 37, 1558-1572.

Tennekes, H., and J. L. Lumley (1972), A First Course in Turbulence, MIT Press, Cambridge, Mass.

Van Ulden, A. P., and J. Wieringa (1996), Atmospheric boundary layer research at Cabauw, Boundary Layer Meteorol., 78, 39-69.

Willis, G. E., and J. W. Deardorff (1974), A laboratory model of the unstable planetary boundary layer, J. Atmos. Sci., 31(5), 1297-1307.

Yamada, T., and G. L. Mellor (1975), A simulation of the Wangara atmospheric boundary layer data, J. Atmos. Sci., 32, 2309-2329.

J. Kleissl, Department of Earth and Environmental Sciences, New Mexico Institute of Mining and Technology, 801 Leroy Place, Socorro, NM 87801, USA. (jan@kleissl.com)

V. Kumar, Department of Geography and Environmental Engineering, Johns Hopkins University, Baltimore, MD 21218, USA. (vijayant@jhu.edu)

C. Meneveau, Department of Mechanical Engineering, Johns Hopkins University, Baltimore, MD 21218, USA. (meneveau@jhu.edu)

M. Parlange, School of Architecture, Civil and Environmental Engineering, Ecole Polytechnique Fédérale de Lausanne, CH-1015 Lausanne, Switzerland. (marc.parlange@epfl.ch) 